\title{
Circle packings, kissing reflection groups and critically fixed anti-rational maps
}

\author{
Russell Lodge $^{1}$, Yusheng Luo ${ }^{2}$ and Sabyasachi Mukherjee ${ }^{\mathbb{D}_{3}}$ \\ ${ }^{1}$ Department of Mathematics and Computer Science, Indiana State University, Terre Haute, 47809, IN, USA; \\ E-mail: russell.lodge@indstate.edu. \\ ${ }^{2}$ Institute for Mathematical Sciences, Stony Brook University, 100 Nicolls Road, Stony Brook, 11794-3660, NY, USA; \\ E-mail: yusheng.s.luo@gmail.com. \\ ${ }^{3}$ School of Mathematics, Tata Institute of Fundamental Research, 1 Homi Bhabha Road, Mumbai, 400005, India; \\ E-mail: sabya@math.tifr.res.in.
}

Received: 17 November 2021; Accepted: 11 December 2021

2020 Mathematics Subject Classification: Primary - 37F10, 37F20, 37F32, 37F34, 51F15; Secondary - 30F40

\begin{abstract}
In this article, we establish an explicit correspondence between kissing reflection groups and critically fixed anti-rational maps. The correspondence, which is expressed using simple planar graphs, has several dynamical consequences. As an application of this correspondence, we give complete answers to geometric mating problems for critically fixed anti-rational maps.
\end{abstract}

\section{Contents}

1 Introduction 1

2 Circle Packings $\quad 7$

3 Kissing Reflection Groups $\quad \mathbf{8}$

3.1 Limit set and domain of discontinuity of kissing reflection groups $\ldots \ldots$. . . . . 8

3.2 Acylindrical kissing reflection groups . . . . . . . . . . . . . . . . . 11

3.3 Deformation spaces of kissing reflection groups . . . . . . . . . . . . . . . . . . . . . .

3.4 Quasi-Fuchsian space and mating locus . . . . . . . . . . . . . . . . 20

3.5 Nielsen maps for kissing reflection groups . . . . . . . . . . . . . . . . . 24

4 Critically Fixed Anti-rational Maps $\quad 25$

4.1 Tischler graph of critically fixed anti-rational maps . . . . . . . . . . . . . . 25

4.2 Constructing critically fixed anti-rational maps from graphs . . . . . . . . . . . . . 27

4.3 Dynamical correspondence . . . . . . . . . . . . . . . . . . . . . . . . . . . . .

4.4 Mating of two anti-polynomials . . . . . . . . . . . . . 33

\section{Introduction}

Ever since Sullivan's translation of Ahlfors' finiteness theorem into a solution of a long-standing open problem on wandering domains in the 1980s [43], many more connections between the theory of Kleinian groups and the study of dynamics of rational functions on $\widehat{\mathbb{C}}$ have been discovered. These

(C) The Author(s), 2022. Published by Cambridge University Press. This is an Open Access article, distributed under the terms of the Creative Commons Attribution licence (https://creativecommons.org/licenses/by/4.0/), which permits unrestricted re-use, distribution, and reproduction in any medium, provided the original work is properly cited. 
analogies between the two branches of conformal dynamics, which are commonly known as Sullivan's dictionary, not only provide a conceptual framework for understanding the connections but motivate research in each field as well.

In this article, we extend this dictionary by establishing a strikingly explicit correspondence between

- Kissing reflection groups: groups generated by reflections along the circles of finite circle packings $\mathcal{P}$ (see Section 3$)$ and

- Critically fixed anti-rational maps: proper anti-holomorphic self-maps of $\widehat{\mathbb{C}}$ with all critical points fixed (see Section 4).

This correspondence can be expressed by a combinatorial model: a simple plane graph $\Gamma$. Throughout this article, a plane graph will mean a planar graph together with an embedding in $\widehat{\mathbb{C}}$. We say that two plane graphs are isomorphic if the underlying graph isomorphism is induced by an orientationpreserving homeomorphism of $\widehat{\mathbb{C}}$. A graph $\Gamma$ is said to be $k$-connected if $\Gamma$ contains more than $k$ vertices and remains connected if any $k-1$ vertices and their corresponding incident edges are removed. Our first result shows the following.

Theorem 1.1. The following three sets are in natural bijective correspondence:

$\circ\{2$-connected simple plane graphs $\Gamma$ with $d+1$ vertices up to isomorphism of plane graphs $\}$,

- $\{$ Kissing reflection groups $G$ of rank $d+1$ with connected limit set up to QC conjugacy\},

- $\{$ Critically fixed anti-rational maps $\mathcal{R}$ of degree d up to Möbius conjugacy\}.

Moreover, if $G_{\Gamma}$ and $\mathcal{R}_{\Gamma}$ correspond to the same plane graph $\Gamma$, then the limit set $\Lambda\left(G_{\Gamma}\right)$ is homeomorphic to the Julia set $\mathcal{J}\left(\mathcal{R}_{\Gamma}\right)$ via a dynamically natural map.

The correspondence between graphs and kissing reflection groups comes from the well-known circle packing theorem (see Theorem 2.1): given a kissing reflection group $G$ with corresponding circle packing $\mathcal{P}$, the plane graph $\Gamma$ associated to $G$ in Theorem 1.1 is the contact graph of the circle packing $\mathcal{P}$. The 2-connectedness condition for $\Gamma$ is equivalent to the connectedness condition for the limit set of $G$ (see Figure 1.1).

On the other hand, given a critically fixed anti-rational map $\mathcal{R}$, we consider the union $\mathscr{T}$ of all fixed internal rays in the invariant Fatou components (each of which necessarily contains a fixed critical point of $\mathcal{R}$ ), known as the Tischler graph (cf. [46]). Roughly speaking, Tischler graphs are to critically fixed (anti-)rational maps what Hubbard trees are to postcritically finite (anti-)polynomials: both are forward invariant graphs containing the postcritical points. We show that the planar dual of $\mathscr{T}$ is a 2 -connected simple plane graph. The plane graph $\Gamma$ we associate to $\mathcal{R}$ in Theorem 1.1 is the planar dual $\mathscr{T}^{\vee}$ of $\mathscr{T}$ (see Figure 1.1).

In the special case when $\Gamma$ is the planar dual of a triangulation of $\widehat{\mathbb{C}}$, the existence of the critically fixed anti-rational map $\mathcal{R}_{\Gamma}$ was proved in [24].

We remark that the correspondence between $G_{\Gamma}$ and $\mathcal{R}_{\Gamma}$ through the plane graph $\Gamma$ is dynamically natural. Indeed, we associate, following [24, §4], a map $\mathcal{N}_{\Gamma}$ to the group $G_{\Gamma}$ with the properties that $\mathcal{N}_{\Gamma}$ and $G_{\Gamma}$ have the same grand orbits (cf. $\left.[33,2]\right)$ and the homeomorphism between $\Lambda\left(G_{\Gamma}\right)$ and $\mathcal{J}\left(\mathcal{R}_{\Gamma}\right)$ conjugates $\mathcal{N}_{\Gamma}$ to $\mathcal{R}_{\Gamma}$. See Subsection 4.3 for more details.

The asymmetry between the QC conjugacies on the group side and Möbius conjugacies on the antirational map side is artificial. Since the dynamical correspondence is between the limit set and the Julia set, Theorem 1.1 yields a bijection between quasiconformal conjugacy classes of kissing reflection groups (of rank $d+1$ ) with connected limit set and hyperbolic components having critically fixed antirational maps (of degree $d$ ) as centres.

\section{The geometric mating problems}

In complex dynamics, polynomial mating is an operation first introduced by Douady in [5] that takes two suitable polynomials $P_{1}$ and $P_{2}$ and constructs a richer dynamical system by carefully pasting together 


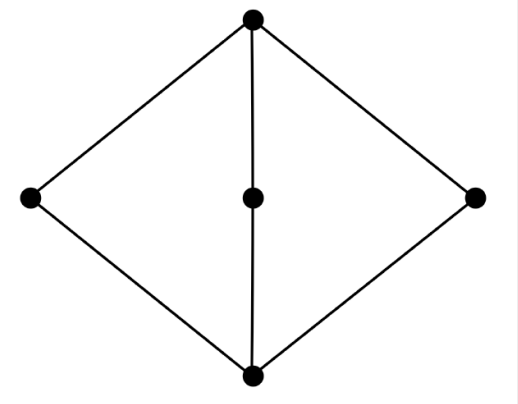

(a) A 2-connected simple plane graph $\Gamma$ with five vertices.

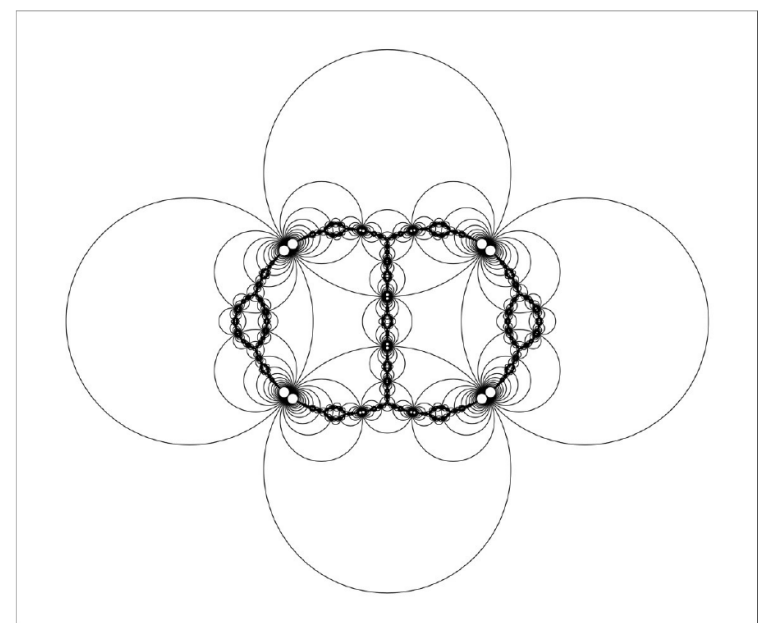

(b) The limit set of a kissing reflection group $G_{\Gamma}$. The four outermost circles together with the central circle form a finite circle packing $\mathcal{P}$ whose contact graph is $\Gamma$. The kissing reflection group $G_{\Gamma}$ is generated by reflections along the five circles in $\mathcal{P}$.

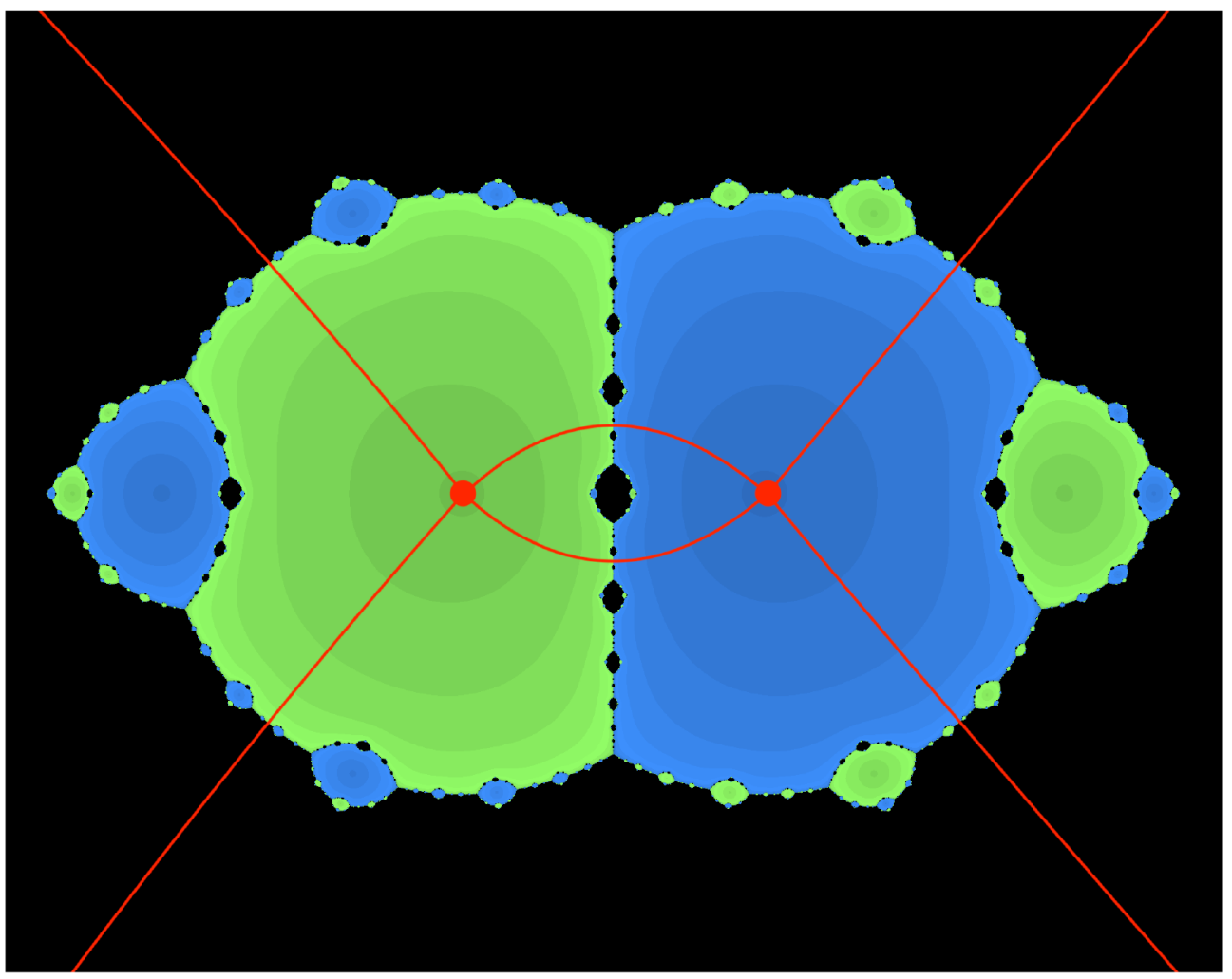

(c) The Julia set of the degree 4 critically fixed anti-rational map $\mathcal{R}_{\Gamma}$. The Tischler graph $\mathscr{T}$, which is drawn schematically in red with two finite vertices and one vertex at infinity, is the planar dual of $\Gamma$.

Figure 1.1. An example of the correspondence. 
the boundaries of their filled Julia sets to obtain a copy of the Riemann sphere, together with a rational map $R$ from this sphere to itself (see Definition 4.16 for the precise formulation). It is natural and important to understand which pairs of polynomials can be mated and which rational maps are matings of two polynomials. The analogous question in the Kleinian group setting can be formulated in terms of Cannon-Thurston maps for degenerations in the quasi-Fuchsian space (see [14] and Subsection 3.3 for related discussions). The correspondence in Theorem 1.1 allows us to explicitly characterise kissing reflection groups and critically fixed anti-rational maps that arise as matings.

We say that a simple plane graph $\Gamma$ with $n$ vertices is outerplanar if it has a face with all $n$ vertices on its boundary. It is said to be Hamiltonian if there exists a Hamiltonian cycle; that is, a closed path visiting every vertex exactly once.

Theorem 1.2. Let $\Gamma$ be a 2-connected simple plane graph. Let $G_{\Gamma}$ and $\mathcal{R}_{\Gamma}$ be a kissing reflection group and a critically fixed anti-rational map associated with $\Gamma$. Then the following hold true:

$\circ \Gamma$ is outerplanar $\Leftrightarrow \mathcal{R}_{\Gamma}$ is a critically fixed anti-polynomial $\Leftrightarrow G_{\Gamma}$ is a function group.

$\circ \Gamma$ is Hamiltonian $\Leftrightarrow \mathcal{R}_{\Gamma}$ is a mating of two polynomials $\Leftrightarrow G_{\Gamma}$ is a mating of two function groups

$\Leftrightarrow G_{\Gamma}$ is in the closure of the quasiconformal deformation space of the regular ideal polygon reflection group.

It is known that a rational map may arise as the geometric mating of more than one pair of polynomials (in other words, the decomposition/unmating of a rational map into a pair of polynomials is not necessarily unique). This phenomenon was first observed in [48] and is referred to as shared matings (see [39]). In our setting, we actually prove that each Hamiltonian cycle of $\Gamma$ gives an unmating of $\mathcal{R}_{\Gamma}$ into two anti-polynomials. Thus, we get many examples of shared matings coming from different Hamiltonian cycles in the associated graphs.

We now address the converse question of mateability in terms of laminations. Let us first note that the question of mateability for kissing reflection groups can be answered using Thurston's double limit theorem and the hyperbolisation theorem. In the reflection group setting, possible degenerations in the quasi-Fuchsian space (of the regular ideal polygon reflection group) are listed by a pair of geodesic laminations on the two conformal boundaries which are invariant under some orientation-reversing involution $\sigma$ (see Subsection 3.3). All of these $\sigma$-invariant laminations turn out to be multicurves on the associated conformal boundaries. A pair of simple closed curves is said to be parallel if they are isotopic under the natural orientation-reversing identification of the two conformal boundary components. A pair of laminations is said to be nonparallel if no two components are parallel. If we lift a multicurve to the universal cover, we get two invariant laminations on the circle. Then they are are nonparallel if and only if the two laminations share no common leaf under the natural identification of the two copies of the circle. Thurston's hyperbolisation theorem asserts that in our setting, the degeneration along a pair of laminations exists if and only if this pair is nonparallel.

For a marked anti-polynomial, we can also associate a lamination via the Bötthcher coordinate at infinity. As before, we say a pair of (anti-polynomial) laminations is nonparallel if they share no common leaf under the natural identification of the two copies of the circle. When we glue two filled Julia sets using the corresponding laminations, the resulting topological space may not be a 2 -sphere. We call this a Moore obstruction. We prove the following more general mateability theorem for postcritically finite anti-polynomials, which in particular answers the question of mateability of two critically fixed anti-polynomials.

Theorem 1.3. Let $P_{1}$ and $P_{2}$ be two marked anti-polynomials of equal degree $d \geq 2$, where $P_{1}$ is critically fixed and $P_{2}$ is postcritically finite, hyperbolic. Then there is an anti-rational map $R$ that is the geometric mating of $P_{1}$ and $P_{2}$ if and only if there is no Moore obstruction.

Consequently, if both $P_{1}$ and $P_{2}$ are critically fixed, then they are geometrically mateable if and only if the corresponding laminations are nonparallel. 


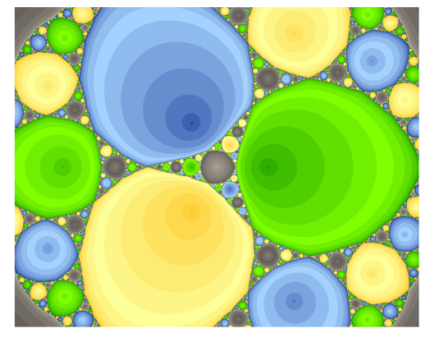

(a) Tetrahedron: $\frac{3 \bar{z}^{2}}{2 \bar{z}^{3}+1}$

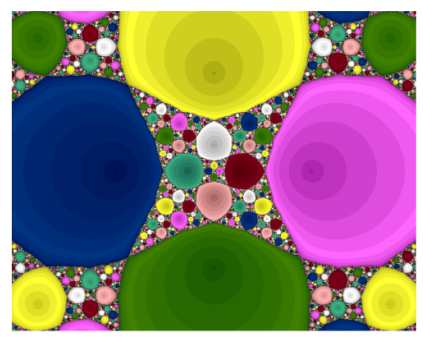

(b) Octahedron: $\frac{5 \bar{z}^{4}+1}{\bar{z}^{5}+5 \bar{z}}$

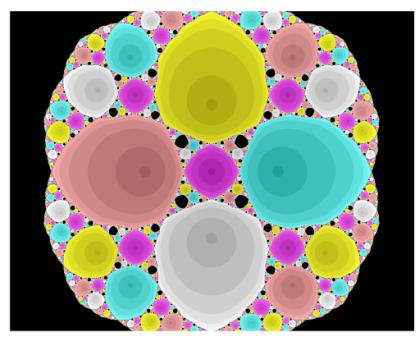

(c) Cube: $\frac{\bar{z}^{7}+7 \bar{z}^{3}}{7 \bar{z}^{4}+1}$

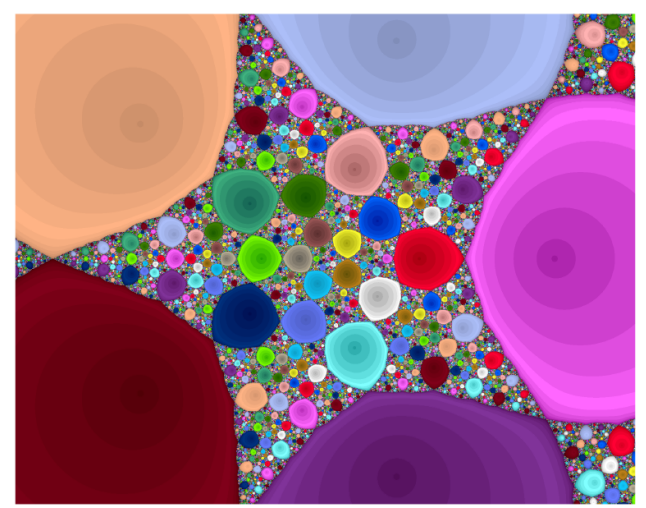

(d) Icosahedron: $\frac{11 \bar{z}^{10}+66 \bar{z}^{5}-1}{\bar{z}^{11}+66 \bar{z}^{6}-11 \bar{z}}$

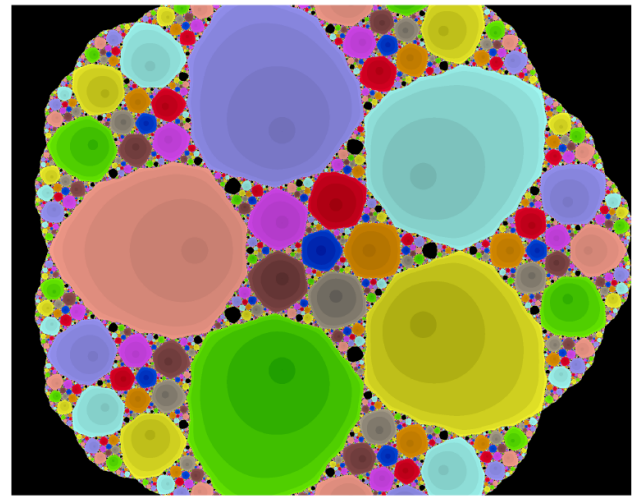

(e) Dodecahedron: $\frac{\bar{z}^{19}-171 \bar{z}^{14}+247 \bar{z}^{9}+57 \bar{z}^{4}}{-57 \bar{z}^{15}+247 \bar{z}^{10}+171 \bar{z}^{5}+1}$

Figure 1.2. Critically fixed anti-rational maps associated to Platonic solids. The Fatou components are coloured according to their grand orbit. The Tischler graph $\mathscr{T}$, which is the planar dual of $\Gamma$, is visible in the figures by connecting the centres of critical fixed Fatou components.

\section{Acylindrical manifolds and gasket sets}

A circle packing is a connected collection of (oriented) circles in $\widehat{\mathbb{C}}$ with disjoint interiors. We say that a closed set $\Lambda$ is a round gasket if

$\circ \Lambda$ is the closure of some infinite circle packing; and

$\circ$ the complement of $\Lambda$ is a union of round disks which is dense in $\widehat{\mathbb{C}}$.

We will call a homeomorphic copy of a round gasket a gasket.

Many examples of kissing reflection groups and critically fixed anti-rational maps have gasket limit sets and Julia sets (see Figure 1.2). The correspondence allows us to classify all of these examples (cf. [17, Theorem 28] and [16]).

Theorem 1.4. Let $\Gamma$ be a 2-connected simple plane graph. Then

$G_{\Gamma}$ has gasket limit set $\Longleftrightarrow \mathcal{R}_{\Gamma}$ has gasket Julia set $\Longleftrightarrow \Gamma$ is 3-connected.

The 3-connectedness for the graph $\Gamma$ also has a characterisation purely in terms of the inherent structure of the hyperbolic 3-manifold associated with $G_{\Gamma}$. Given a kissing reflection group $G_{\Gamma}$, the index 2 subgroup $\widetilde{G}_{\Gamma}$ consisting of orientation-preserving elements is a Kleinian group. We say that $G_{\Gamma}$ is acylindrical if the hyperbolic 3-manifold for $\widetilde{G}_{\Gamma}$ is acylindrical (see Subsection 3.2 for the precise definitions). We show the following.

Theorem 1.5. Let $\Gamma$ be a 2-connected simple plane graph. Then

$G_{\Gamma}$ is acylindrical $\Longleftrightarrow \Gamma$ is 3-connected. 


\section{Parameter space implications of the dictionary}

We now briefly mention some natural questions regarding parameter spaces of anti-rational maps raised by the aforementioned dictionary between kissing reflection groups and critically fixed anti-rational maps.

Acylindrical manifolds play an important role in 3-dimensional geometry and topology. Relevant to our discussion, Thurston proved that the deformation space of an acylindrical 3-manifold is bounded [45] (this is famously known as Thurston's compactness theorem). The analogue of deformation spaces in holomorphic dynamics is hyperbolic components. The analogy established in Theorems 1.4 and 1.5 leads one to ask whether there is a counterpart of Thurston's compactness theorem for hyperbolic components of critically fixed anti-rational maps with gasket Julia sets (see [29, Question 5.3] for the corresponding question in the convex cocompact setting). In a forthcoming paper, a suitably interpreted boundedness result will be proved for the hyperbolic components under consideration [23].

In [13], Hatcher and Thurston studied the topology of the moduli space of marked circle packings with $n$ circles in $\mathbb{C}$. In particular, they showed that the map that sends a marked circle packing to the centres of the circles yields a homotopy equivalence between the moduli space of marked circle packings and the configuration space of $n$ marked points in $\mathbb{C}$. Motivated by this statement, one may ask whether the union of the closures of the hyperbolic components of degree $d$ critically fixed anti-rational maps have a nontrivial topology. This question will be answered affirmatively in a future work, drawing exact parallels between contact structures of quasiconformal deformation spaces of kissing reflection groups and the corresponding hyperbolic component closures [23].

We now summarise these correspondences in the following table. ${ }^{1}$

\begin{tabular}{lll}
\hline Simple plane graph & Kleinian groups & Complex dynamics \\
\hline Connected & Kissing reflection group & - \\
2-connected & Connected limit set & Critically fixed anti-rational map \\
3-connected/polyhedral & Gasket limit set/acylindrical/bounded QC & Gasket Julia set/bounded pared deformation space \\
& deformation space & Critically fixed anti-polynomial \\
Outerplanar & Function kissing reflection group & Mating of two anti-polynomials \\
Hamiltonian & Closure of quasi-Fuchsian space & A pair of nonparallel anti-polynomial laminations \\
A marked Hamiltonian cycle & A pair of nonparallel multicurves & The hyperbolic component $\mathcal{H}_{\Gamma_{1}}$ bifurcates to $\mathcal{H}_{\Gamma_{2}}$ \\
A graph $\Gamma_{1}<\Gamma_{2}$ & The QC deformation space & \\
& $\mathcal{Q C}\left(\Gamma_{2}\right) \subseteq \partial \mathcal{Q C}\left(\Gamma_{1}\right)$ & \\
\hline
\end{tabular}

\section{Notes and references}

Aspects of Sullivan's dictionary were already anticipated by Fatou [8, p. 22]. Part of the correspondence in Theorem 1.1 was recently established in [24] where the Tischler graphs were assumed to be triangulations. A classification of critically fixed anti-rational maps has also been obtained in [9]. There is also a connection between Kleinian reflection groups, anti-rational maps and Schwarz reflections of quadrature domains explained in [21, 24, 19, 20]. In the holomorphic setting, critically fixed rational maps have been studied in $[4,11]$.

Many critically fixed anti-rational maps have large symmetry groups. The examples corresponding to the five platonic solids are listed in Figure 1.2. The counterparts in the holomorphic setting were constructed in [7] (see also [3, 12]).

The connections between number-theoretic problems for circle packings and equidistribution results for Kleinian groups can be found in [18, 34, 17].

${ }^{1}$ Here we have also included the parameter space implications, which will be studied in a subsequent paper. 


\section{Some questions}

The dictionary between kissing reflection groups and critically fixed anti-rational maps raises some natural questions. Let us fix a 2-connected simple plane graph $\Gamma$.

○ Although the limit and Julia sets $\Lambda\left(G_{\Gamma}\right)$ and $\mathcal{J}\left(\mathcal{R}_{\Gamma}\right)$ are homeomorphic, the dynamically natural homeomorphism is not a quasisymmetry because it sends parabolic fixed points to repelling ones. In fact, we believe that there is no quasiconformal homeomorphism of the sphere carrying one to the other. In the case when $\Gamma$ is 3-connected, this follows from the observation that the boundaries of two components of the domain of discontinuity of $G_{\Gamma}$ touch at a cusp (with zero angle), while the boundaries of two Fatou components of $\mathcal{R}_{\Gamma}$ touch at a repelling (pre-) fixed point (with a positive angle). However, in general, we do not know how to rule out the existence of 'exotic' quasiconformal homeomorphisms between $\Lambda\left(G_{\Gamma}\right)$ and $\mathcal{J}\left(\mathcal{R}_{\Gamma}\right)$. Moreover, since the quasisymmetry group of a fractal is a quasiconformal invariant, it will be interesting to know the quasisymmetry groups of $\Lambda\left(G_{\Gamma}\right)$ and $\mathcal{J}\left(\mathcal{R}_{\Gamma}\right)$. Note that according to [24], the quasisymmetry groups of these two fractals are isomorphic when the dual of $\Gamma$ is an unreduced triangulation.

- Our proof of the existence of a homeomorphism between $\Lambda\left(G_{\Gamma}\right)$ and $\mathcal{J}\left(\mathcal{R}_{\Gamma}\right)$ only makes use of the conformal dynamics of the group and the anti-rational map on $\widehat{\mathbb{C}}$. One would like to know whether there is a direct 3-dimensional interpretation of this result.

\section{Structure of the article}

We collect various known circle packing theorems in Section 2. Based on this, we prove the connection between kissing reflection groups and simple plane graphs in Section 3. In particular, the group part of Theorem 1.1, Theorem 1.2, Theorem 1.4 and Theorem 1.5 is proved in Proposition 3.4, Propositions 3.18, 3.20, 3.21, Proposition 3.10 and Proposition 3.6, respectively.

Critically fixed anti-rational maps are studied in Section 4. The anti-rational map part of Theorem 1.1 is proved in Proposition 4.10. Once this is established, the anti-rational map part of Theorem 1.2 and Theorem 1.4 follow from their group counterparts as explained in Corollary 4.17. Theorem 1.3 is proved in Proposition 4.23 and Corollary 4.24.

\section{Circle Packings}

In this article, a circle packing $\mathcal{P}$ is a connected finite collection of (oriented) circles in $\widehat{\mathbb{C}}$ with disjoint interiors. Unless stated otherwise, the circle packings in this article are assumed to contain at least three circles. The combinatorics of configuration of a circle packing can be described by its contact graph $\Gamma$ : we associate a vertex to each circle and two vertices are connected by an edge if and only if the two associated circles intersect. The embedding of the circles in $\widehat{\mathbb{C}}$ determines the isomorphism class of its contact graph as a plane graph. It can also be checked easily that the contact graph of a circle packing is simple. This turns out to be the only constraint for the graph (See [44, Chapter 13]).

Theorem 2.1. (Circle Packing Theorem). Every connected simple plane graph is isomorphic to the contact graph of some circle packing.

The definition of contact graphs can be easily generalised to an infinite collection of circles with disjoint interiors. In this article, an infinite circle packing $\mathcal{P}$ is an infinite collection of (oriented) circles in $\widehat{\mathbb{C}}$ with disjoint interiors, whose contact graph is connected.

\section{k-connected graphs}

A graph $\Gamma$ is said to be $k$-connected if $\Gamma$ contains more than $k$ vertices and remains connected if any $k-1$ vertices and their corresponding incident edges are removed. A graph is $\Gamma$ is said to be polyhedral 
if $\Gamma$ is the 1-skeleton of a convex polyhedron. According to Steinitz's theorem, a graph is polyhedral if and only if it is 3-connected and planar.

Given a polyhedral graph, we have a stronger version of the circle packing theorem [40] (cf. midsphere for canonical polyhedron).

Theorem 2.2. (Circle Packing Theorem for polyhedral graphs). Suppose $\Gamma$ is a polyhedral graph; then there is a pair of circle packings whose contact graphs are isomorphic to $\Gamma$ and its planar dual. Moreover, the two circle packings intersect orthogonally at their points of tangency.

This pair of circle packings is unique up to Möbius transformations.

\section{Marked contact graphs}

In many situations, it is better to work with a marking on the graph as well as the circle packing. A marking of a graph $\Gamma$ is a choice of the graph isomorphism

$$
\phi: \mathscr{G} \longrightarrow \Gamma
$$

where $\mathscr{G}$ is the underlying abstract graph of $\Gamma$. We will refer to the pair $(\Gamma, \phi)$ as a marked graph.

Given two marked plane graphs $\left(\Gamma_{1}, \phi_{1}\right)$ and $\left(\Gamma_{2}, \phi_{2}\right)$ with the same underlying abstract graph $\mathscr{G}$, we say that they are equivalent if $\phi_{2} \circ \phi_{1}^{-1}: \Gamma_{1} \longrightarrow \Gamma_{2}$ is an isomorphism of plane graphs.

Similarly, a circle packing $\mathcal{P}$ is said to be marked if the associated contact graph is marked.

\section{Kissing Reflection Groups}

Let $\Gamma$ be a marked connected simple plane graph. By the circle packing theorem, $\Gamma$ is (isomorphic to) the contact graph of some marked circle packing

$$
\mathcal{P}=\left\{C_{1}, \ldots, C_{n}\right\}
$$

We define the kissing reflection group associated to this circle packing $\mathcal{P}$ as

$$
G_{\mathcal{P}}:=\left\langle g_{1}, \ldots, g_{n}\right\rangle,
$$

where $g_{i}$ is the reflection along the circle $C_{i}$.

We denote the group of all Möbius automorphisms of $\widehat{\mathbb{C}}$ by $\operatorname{Aut}^{+}(\widehat{\mathbb{C}})$ and the group of all Möbius and anti-Möbius automorphisms of $\widehat{\mathbb{C}}$ by $\operatorname{Aut}^{ \pm}(\widehat{\mathbb{C}})$. Note that since a kissing reflection group is a discrete subgroup of $\operatorname{Aut}^{ \pm}(\widehat{\mathbb{C}})$, definitions of limit set and domain of discontinuity can be easily extended to kissing reflection groups. We shall use $\widetilde{G}_{\mathcal{P}}$ to denote the index 2 subgroup of $G_{\mathcal{P}}$ consisting of orientation-preserving elements. Note that $\widetilde{G}_{\mathcal{P}}$ lies on the boundary of Schottky groups.

We remark that if $\mathcal{P}^{\prime}$ is another circle packing realising $\Gamma$, since the graph $\Gamma$ is marked, there is a canonical identification of the circle packing $\mathcal{P}^{\prime}$ with $\mathcal{P}$. This gives a canonical isomorphism between the kissing reflection groups $G_{\mathcal{P}^{\prime}}$ and $G_{\mathcal{P}}$, which is induced by a quasiconformal map. We refer to $\Gamma$ as the contact graph associated to $G_{\mathcal{P}}$.

\subsection{Limit set and domain of discontinuity of kissing reflection groups}

Let $\mathcal{P}=\left\{C_{1}, \ldots, C_{n}\right\}$ be a marked circle packing and $D_{i}$ be the associated open disks for $C_{i}$. Let $P$ be the set consisting of points of tangency for the circle packing $\mathcal{P}$. Let

$$
\Pi=\widehat{\mathbb{C}} \backslash\left(\bigcup_{i=1}^{n} D_{i} \cup P\right) .
$$


Then $\Pi$ is a fundamental domain of the action of $G_{\mathcal{P}}$ on the domain of discontinuity $\Omega\left(G_{\mathcal{P}}\right)$. Denote $\Pi=\bigcup_{i=1}^{k} \Pi_{i}$ where $\Pi_{i}$ is a component of $\Pi$. Note that when the limit set of $G_{\mathcal{P}}$ is connected, each component of the domain of discontinuity is simply connected (that is, a conformal disk) and each component $\Pi_{i}$ is a closed ideal polygon in the corresponding component of the domain of discontinuity bounded by arcs of finitely many circles in the circle packing.

Since $G_{\mathcal{P}}$ is a free product of finitely many copies of $\mathbb{Z} / 2 \mathbb{Z}$, each element $g \in G_{\mathcal{P}}$ admits a shortest expression $g=g_{i_{1}} \ldots g_{i_{l}}\left(i_{r} \neq i_{r+1}\right.$, for $\left.r \in\{1, \cdots, l-1\}\right)$ with respect to the standard generating set $S=\left\{g_{1}, \ldots, g_{n}\right\}$. The integer $l$ is called the length of the group element $g$, thought of as a word in terms of the generators.

The following lemma follows directly by induction.

Lemma 3.1. Let $g=g_{i_{1}} \ldots g_{i_{l}}$ be an element of length $l$, then $g \cdot \Pi \subseteq \bar{D}_{i_{1}}$.

We set $\Pi^{1}:=\bigcup_{i=1}^{n} g_{i} \cdot \Pi$ and $\Pi^{j+1}=\bigcup_{i=1}^{n} g_{i} \cdot\left(\Pi^{j} \backslash \bar{D}_{i}\right)$. For consistency, we also set $\Pi^{0}:=\Pi$. We call $\Pi^{l}$ the tiling of level $l$. The following lemma justifies this terminology.

Lemma 3.2.

$$
\Pi^{l}=\bigcup_{|g|=l} g \cdot \Pi .
$$

Proof. We will prove this by induction. The base case is satisfied by the definition of $\Pi^{1}$. Assume that $\Pi^{j}=\bigcup_{|g|=j} g \cdot \Pi$. Let $g=g_{i_{1}} g_{i_{2}} \ldots g_{i_{j+1}}$ be of length $j+1$. Note that $i_{1} \neq i_{2}$. By Lemma 3.1, $g_{i_{2}} g_{i_{3}} \ldots g_{i_{j+1}} \cdot \Pi$ does not intersect $\bar{D}_{i_{1}}$; thus, $g \cdot \Pi \subseteq g_{i_{1}} \cdot\left(\Pi^{j} \backslash \bar{D}_{i_{1}}\right)$. So $\bigcup_{|g|=j+1} g \cdot \Pi \subseteq \Pi^{j+1}$. The reverse inclusion can be proved similarly.

Since the domain of discontinuity is the union of tiles of all levels, Lemma 3.2 implies that

$$
\Omega\left(G_{\mathcal{P}}\right)=\bigcup_{g \in G_{\mathcal{P}}} g \cdot \Pi=\bigcup_{i=0}^{\infty} \Pi^{i} .
$$

Similarly, we set $\mathcal{D}^{i}=\widehat{\mathbb{C}} \backslash \bigcup_{j=0}^{i} \Pi^{j}$. We remark that $\mathcal{D}^{i}$ is neither open nor closed: it is a finite union of open disks together with the orbit of $P$ under the group elements of length up to $i$ on the boundaries of these disks.

Let $\overline{\mathcal{D}^{i}}$ be its closure. Note that each $\overline{\mathcal{D}^{i}}$ is a union of closed disks for some (possibly disconnected) finite circle packing. Indeed,

$$
\overline{\mathcal{D}^{0}}=\overline{\widehat{\mathbb{C}} \backslash \Pi^{0}}=\bigcup_{i=1}^{n} \bar{D}_{i}
$$

is the union of the closed disks corresponding to the original circle packing $\mathcal{P}$. By induction, we have that at level $i+1$,

$$
\overline{\mathcal{D}^{i+1}}=\bigcup_{j=1}^{n} g_{j} \cdot \overline{\mathcal{D}^{i} \backslash \bar{D}_{j}}
$$

is the union of the images of the level $i$ disks outside of $\bar{D}_{j}$ under $g_{j}$.

We also note that the sequence $\overline{\mathcal{D}}^{i}$ is nested and thus the limit set

$$
\Lambda\left(G_{\mathcal{P}}\right)=\bigcap_{i=0}^{\infty} \mathcal{D}^{i}=\bigcap_{i=0}^{\infty} \overline{\mathcal{D}^{i}} .
$$

Therefore, we have the following expansive property of the group action on $\Lambda\left(G_{\mathcal{P}}\right)$. 


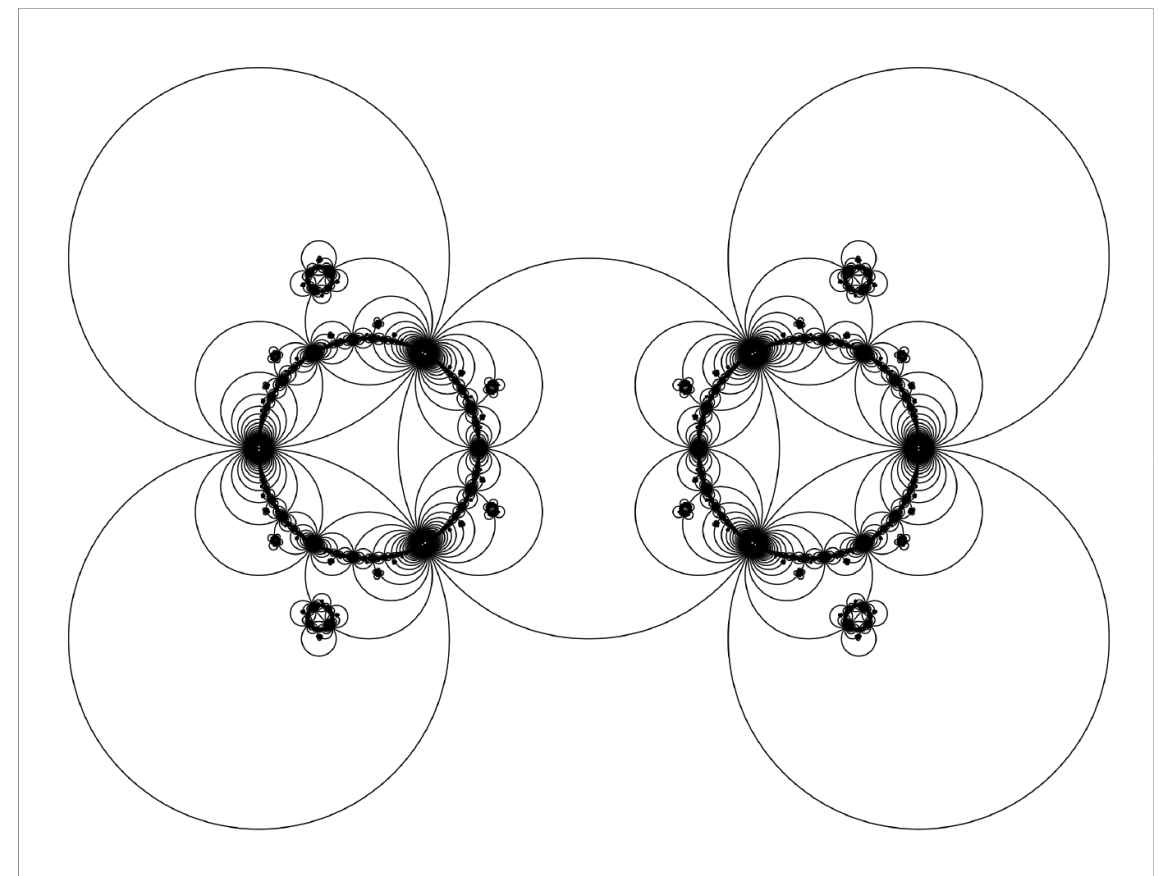

Figure 3.1. A disconnected limit set for a kissing reflection group $G$ with non-2-connected contact graph. $G$ is generated by reflections along the five visible large circles in the figure.

Lemma 3.3. Let $r_{n}$ be the maximum spherical diameter of the disks in $\mathcal{D}^{n}$. Then $r_{n} \rightarrow 0$.

Proof. Otherwise, we can construct a sequence of nested disks of radius bounded from below implying that the limit set contains a disk, which is a contradiction.

We now prove the group part of Theorem 1.1.

Proposition 3.4. The kissing reflection group $G_{\mathcal{P}}$ has connected limit set if and only if the contact graph $\Gamma$ of $\mathcal{P}$ is 2-connected.

Proof. If $\Gamma$ is not 2-connected, then there exists a circle (say $C_{1}$ ) such that the circle packing becomes disconnected once we remove it. Then we see $\mathcal{D}^{1} \subseteq \widehat{\mathbb{C}}$ is disconnected by Equation 3.1. This forces the limit set to be disconnected as well (see Figure 3.1).

On the other hand, if $\Gamma$ is 2-connected, then $\mathcal{D}^{1}$ is connected by Equation 3.1. Now by induction and Equation 3.1 again, $\mathcal{D}^{i}$ is connected for all $i$. Thus, $\Lambda\left(G_{\mathcal{P}}\right)=\bigcap_{i=0}^{\infty} \mathcal{D}^{i}$ is also connected.

Proposition 3.4 and the definition of kissing reflection groups show that the association of a 2-connected simple plane graph with a kissing reflection group with connected limit set is well defined and surjective. To verify that this is indeed injective, we remark that if $\mathcal{P}$ and $\mathcal{P}^{\prime}$ are two circle packings associated to two contact graphs that are nonisomorphic as plane graphs, then the closures of the fundamental domains $\bar{\Pi}$ and $\overline{\Pi^{\prime}}$ are not homeomorphic. Note that the touching patterns of different components of $\Pi$ or $\Pi^{\prime}$ completely determine the structures of the pairing cylinders of the associated 3 -manifolds at the cusps (See $[25, \S 2.6]$ ). This means that the conformal boundaries of $\mathbb{H}^{3} / \widetilde{G}_{\mathcal{P}}$ and $\mathbb{H}^{3} / \widetilde{G}_{\mathcal{P}^{\prime}}$ with the pairing cylinder structures are not the same. Thus, the two kissing reflection groups $G_{\mathcal{P}}$ and $G_{\mathcal{P}^{\prime}}$ are not quasiconformally isomorphic. 


\subsection{Acylindrical kissing reflection groups}

Recall that $\widetilde{G}_{\mathcal{P}}$ is the index 2 subgroup of $G_{\mathcal{P}}$ consisting of orientation-preserving elements. We set

$$
\mathcal{M}\left(G_{\mathcal{P}}\right):=\mathbb{H}^{3} \cup \Omega\left(G_{\mathcal{P}}\right) / \widetilde{G}_{\mathcal{P}}
$$

to be the associated 3-manifold with boundary. Note that the boundary

$$
\partial \mathcal{M}\left(G_{\mathcal{P}}\right)=\Omega\left(G_{\mathcal{P}}\right) / \widetilde{G}_{\mathcal{P}}
$$

is a finite union of punctured spheres. Each punctured sphere corresponds to the double of a component of $\Pi$.

Let $F$ be a face of $\Gamma$. Then it corresponds to a component $\Pi_{F}$ of $\Pi$, which also corresponds to a component $R_{F}$ of $\partial \mathcal{M}\left(G_{\mathcal{P}}\right)$. More precisely, there is a unique component $\Omega_{F}$ of $\Omega\left(G_{\mathcal{P}}\right)$ containing $\Pi_{F}$ and

$$
R_{F} \cong \Omega_{F} / \operatorname{stab}\left(\Omega_{F}\right)
$$

where $\operatorname{stab}\left(\Omega_{F}\right)$ is the stabiliser of $\Omega_{F}$ in $\widetilde{G}_{\mathcal{P}}$.

A compact 3-manifold $M^{3}$ with boundary is called acylindrical if $M^{3}$ contains no essential cylinders and is boundary incompressible. Here an essential cylinder $C$ in $M^{3}$ is a closed cylinder $C$ such that $C \cap \partial M^{3}=\partial C$, the boundary components of $C$ are not homotopic to points in $\partial M^{3}$ and $C$ is not homotopic into $\partial M^{3} . M^{3}$ is said to be boundary incompressible if the inclusion $\pi_{1}(R) \hookrightarrow \pi_{1}\left(M^{3}\right)$ is injective for every component $R$ of $\partial M^{3}$. (We refer the reader to [26, §3.7, §4.7] for detailed discussions.)

Our manifold $\mathcal{M}\left(G_{\mathcal{P}}\right)$ is not a compact manifold as there are parabolic elements (cusps) in $\widetilde{G}_{\mathcal{P}}$. Thurston [45] introduced the notion of pared manifolds to work with Kleinian groups with parabolic elements. In our setting, we can also use an equivalent definition without introducing pared manifolds. To start the definition, we note that for a geometrically finite group, associated with the conjugacy class of a rank 1 cusp, there is a pair of punctures $p_{1}, p_{2}$ on $\partial M^{3}$. If $c_{1}, c_{2}$ are small circles in $\partial M$ retractable to $p_{1}, p_{2}$, then there is a pairing cylinder $C$ in $M^{3}$, which is a cylinder bounded by $c_{1}$ and $c_{2}$ (see [25, $\S 2.6],[26$, p. 125]).

Definition 3.5. A kissing reflection group $G_{\mathcal{P}}$ is said to be acylindrical if $\mathcal{M}\left(G_{\mathcal{P}}\right)$ is boundary incompressible and every essential cylinder is homotopic to a pairing cylinder.

Note that $\mathcal{M}\left(G_{\mathcal{P}}\right)$ is boundary incompressible if and only if each component of $\Omega\left(G_{\mathcal{P}}\right)$ is simply connected if and only if the limit set $\Lambda\left(G_{\mathcal{P}}\right)$ is connected. We also note that the acylindrical condition is a quasiconformal invariant and hence does not depend on the choice of the circle packing $\mathcal{P}$ realising a simple connected plane graph $\Gamma$. In the remainder of this section, we shall prove the following characterisation of acylindrical kissing Kleinian reflection groups.

Proposition 3.6. The kissing reflection group $G_{\mathcal{P}}$ is acylindrical if and only if the contact graph $\Gamma$ of $\mathcal{P}$ is 3-connected.

This proposition will be proved after the following lemmas. Let $\Gamma$ be a 2-connected simple plane graph and $\mathcal{P}=\left\{C_{1}, \ldots, C_{n}\right\}$ be a realisation of $\Gamma$. Let $G_{\mathcal{P}}$ be the kissing reflection group, with generators $g_{1}, \ldots, g_{n}$ given by reflections along $C_{1}, \ldots, C_{n}$. Note that a face $F$ of $\Gamma$ corresponds to a component $R_{F}$ of $\partial \mathcal{M}\left(G_{\mathcal{P}}\right)$.

Any two nonadjacent vertices $v, w$ of the face $F$ give rise to an essential simple closed curve $\widetilde{\gamma}_{v w}^{F}$ on $R_{F}$ (a simple closed curve on a surface is essential if it is not homotopic to a point or a puncture). More precisely, let $g_{v}, g_{w}$ be the reflections associated to the two vertices; then $g_{v} g_{w} \in \operatorname{stab}\left(\Omega_{F}\right)$ is a loxodromic element under the uniformisation of $R_{F}$ which gives the simple closed curve $\widetilde{\gamma}_{v w}^{F}$ on $R_{F}$. 


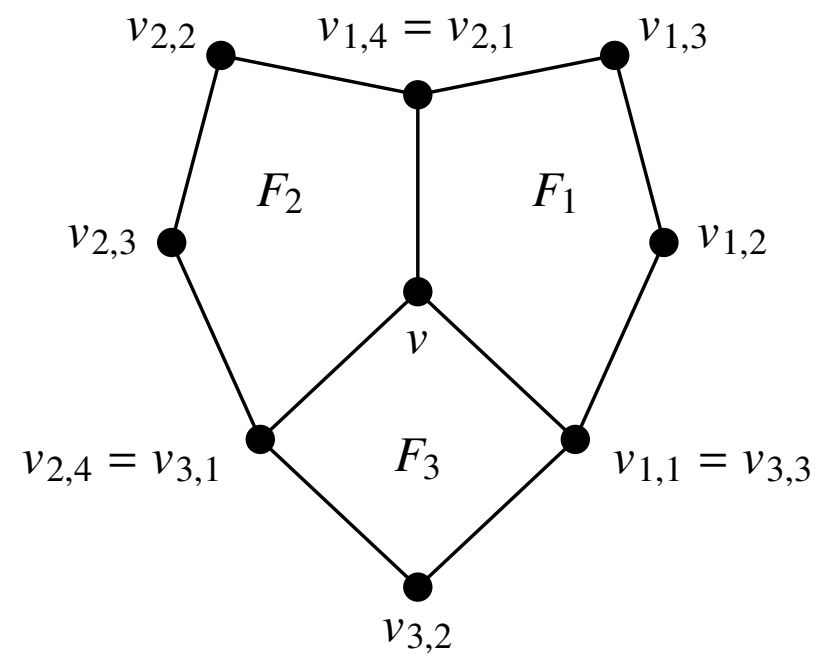

Figure 3.2. A schematic of the potentially non-simple cycle around $v$.

Note that $g_{v} g_{w}$ itself may not be loxodromic as the vertices $v, w$ may be adjacent in some other faces. If this is the case, then we have an accidental parabolic element (see [26, p. 198, Section 3, Problem 17]).

We first prove the following graph-theoretic lemma.

Lemma 3.7. Let $\Gamma$ be a 2-connected simple plane graph. If $\Gamma$ is not 3-connected, then there exist two vertices $v, w$ so that $v, w$ lie on the intersection of the boundaries of two faces $F_{1}$ and $F_{2}$. Moreover, they are nonadjacent for at least one of the two faces.

Proof. As $\Gamma$ is not 3-connected, there exist two vertices $v, w$ so that $\Gamma \backslash\{v, w\}$ is disconnected. Let $F_{1}, \ldots, F_{k}$ be the list of faces that contain $v$ as a vertex. Since $\Gamma$ is plane, we may assume that the faces $F_{i}$ are ordered around $v$ counterclockwise. Since $\Gamma$ is plane and 2-connected, each face $F_{i}$ is a Jordan domain. Let $v_{i, 0}=v, v_{i, 1}, \ldots, v_{i, j_{i}}$ be the vertices of $F_{i}$ ordered counterclockwise. Since the faces $F_{i}$ are ordered counterclockwise, we have that $v_{i, j_{i}}=v_{i+1,1}$. We remark that there might be additional identifications. Then

$$
v_{1,1} \rightarrow v_{1,2} \rightarrow \ldots \rightarrow v_{1, j_{1}}\left(=v_{2,1}\right) \rightarrow v_{2,2} \rightarrow \ldots \rightarrow v_{k, j_{k}}=v_{1,1}
$$

form a (potentially non-simple) cycle $C$ (see Figure 3.2). Since $\Gamma$ is 2-connected, $\Gamma \backslash\{w\}$ is connected. Thus, in particular, any vertex $p$ is connected to $C \backslash\{w\}$ in $\Gamma \backslash\{w\}$. Thus, if $w \notin C$ or $w$ only appears once in $C$, then $C \backslash\{w\}$ is connected. This would imply that $\Gamma \backslash\{v, w\}$ is connected, which is a contradiction. Therefore, $w$ must appear at least twice in the cycle $C$. Since each face is a Jordan domain, $w$ must appear on the boundaries of at least two faces $F_{i_{1}}$ and $F_{i_{2}}$.

Since $\Gamma$ is simple, $w$ is adjacent to $v$ in at most two faces, in which case $w=v_{i, j_{i}}=v_{i+1,1}$; that is, it contributes to only one point in $C$. Therefore, there exists a face on which $w$ is not adjacent to $v$. This proves the lemma.

We can now prove one direction of Proposition 3.6.

Lemma 3.8. If $G_{\mathcal{P}}$ is acylindrical, then $\Gamma$ is 3-connected.

Proof. Note that $\Gamma$ must be 2-connected as $\Lambda\left(G_{\mathcal{P}}\right)$ is connected (by the boundary incompressibility condition). We will prove the contrapositive and assume that $\Gamma$ is not 3 -connected. Let $v, w$ be the two vertices given by Lemma 3.7. There are two cases. 


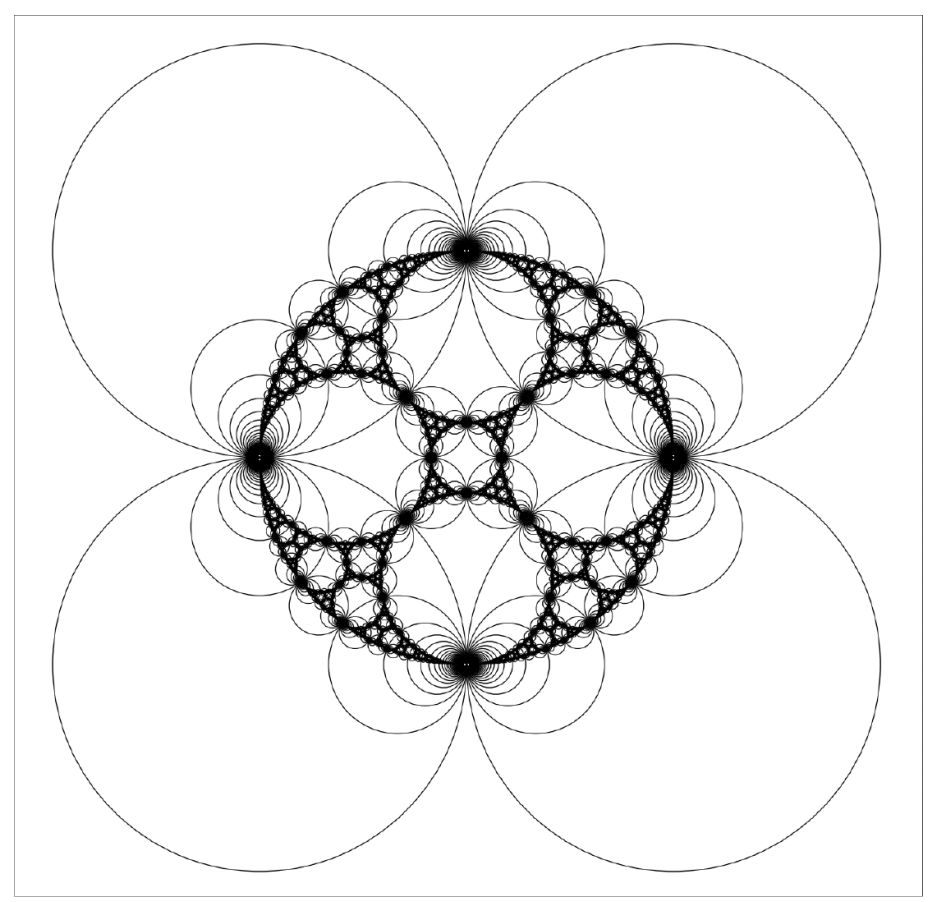

Figure 3.3. The limit set of a kissing reflection group $G$ with a 3-connected contact graph.

If $v, w$ are nonadjacent vertices in two faces $F_{1}, F_{2}$, then $g_{v} g_{w}$ gives a pair of essential simple closed curves on $R_{F_{1}}$ and $R_{F_{2}}$ in $\partial \mathcal{M}\left(G_{\mathcal{P}}\right)$. This pair bounds an essential cylinder (see the Cylinder Theorem in $[26, \S 3.7]$ ), which is not homotopic to a pairing cylinder of two punctures (see $g_{C} g_{C^{\prime \prime}}$ in Figure 3.4).

If $v, w$ are nonadjacent vertices in $F_{1}$ but adjacent vertices in $F_{2}$, then $g_{v} g_{w}$ corresponds to an essential simple closed curve in $R_{F_{1}}$ and a simple closed curve homotopic to a puncture in $R_{F_{2}}$. Then $g_{v} g_{w}$ is an accidental parabolic and the two curves bound an essential cylinder which is not homotopic to a pairing cylinder (see $g_{C} g_{C^{\prime}}$ in Figure 3.4).

Therefore, in either case, $G_{\mathcal{P}}$ is cylindrical.

\section{Gasket limit set}

Recall that a closed set $\Lambda$ is a round gasket if

$\circ \Lambda$ is the closure of some infinite circle packing; and

$\circ$ the complement of $\Lambda$ is a union of round disks which is dense in $\widehat{\mathbb{C}}$.

We call a homeomorphic copy of a round gasket a gasket. See Section 2 for our definition of infinite circle packings.

If $\Gamma$ is 3-connected, then $\Gamma$ is a polyhedral graph. Let $\Gamma^{\vee}$ be the planar dual of $\Gamma$. Then Theorem 2.2 gives a (unique) pair of circle packings $\mathcal{P}$ and $\mathcal{P}^{\vee}$ whose contact graphs are isomorphic to $\Gamma$ and $\Gamma^{\vee}$ (respectively) as plane graphs such that $\mathcal{P}$ and $\mathcal{P}^{\vee}$ intersect orthogonally at their points of tangency (see Figure 3.3). Let $G_{\mathcal{P}}$ be the kissing reflection group associated with $\mathcal{P}$. Since the circle packing $\mathcal{P}^{\vee}$ is dual to $\mathcal{P}$, we have that

$$
\bigcup_{g \in G_{\mathcal{P}}} \bigcup_{C \in \mathcal{P}^{\vee}} g \cdot C
$$




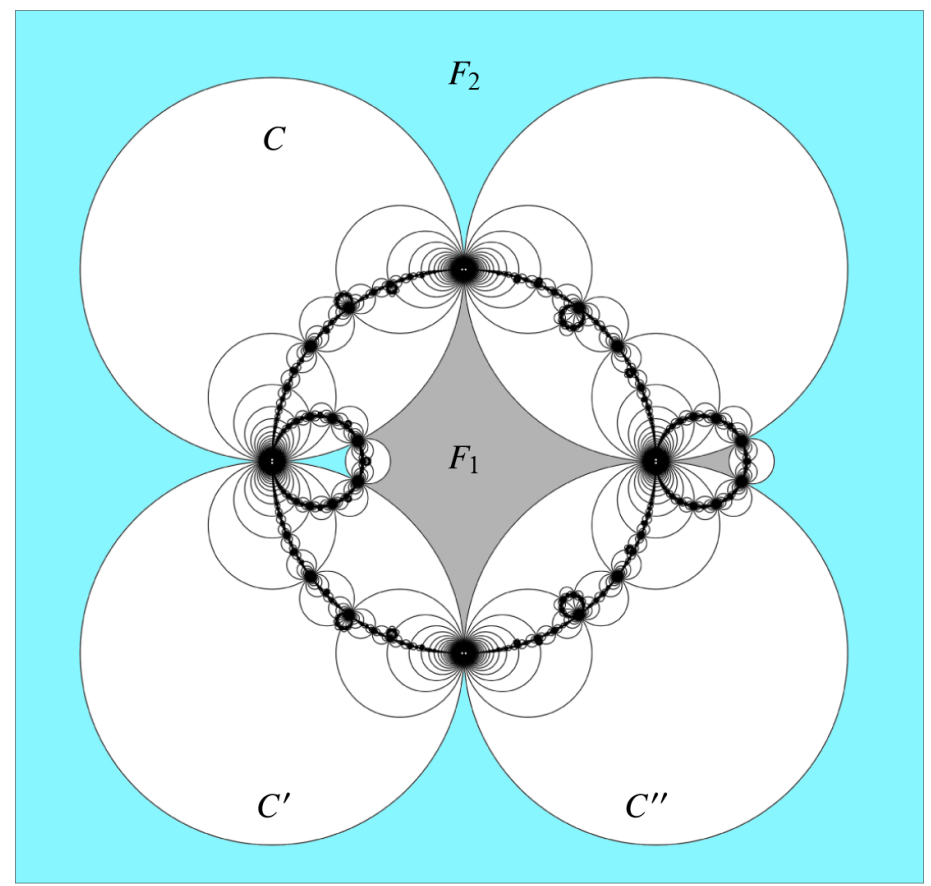

Figure 3.4. The limit set of a kissing reflection group $G$ with Hamiltonian but non-3-connected contact graph. The unique Hamiltonian cycle of the associated contact graph $\Gamma$ divides the fundamental domain $\Pi(G)$ into two parts $\Pi^{ \pm}$, which are shaded in grey and blue. With appropriate markings, $G$ is the mating of two copies of the group H shown in Figure 3.6.

is an infinite circle packing and the limit set is the closure

$$
\Lambda\left(G_{\mathcal{P}}\right)=\overline{\bigcup_{g \in G_{\mathcal{P}}} \bigcup_{C \in \mathcal{P}^{\vee}} g \cdot C .}
$$

Since $\Lambda\left(G_{\mathcal{P}}\right)$ is nowhere dense and the complement is a union of round disks, we conclude that $\Lambda\left(G_{\mathcal{P}}\right)$ is a round gasket.

Note that each component of $\Omega\left(G_{\mathcal{P}}\right)$ is of the form $g \cdot D$ where $g \in G_{\mathcal{P}}$ and $D$ is a disk in the dual circle packing $\mathcal{P}^{\vee}$. By induction, we have the following.

Lemma 3.9. If $\Gamma$ is 3-connected, then the closure of any two different components of $\Omega\left(G_{\mathcal{P}}\right)$ only intersect at cusps.

We have the following characterisation of gasket limit set for kissing reflection groups.

Proposition 3.10. Let $\Gamma$ be a simple plane graph; then $\Lambda\left(G_{\mathcal{P}}\right)$ is a gasket if and only if $\Gamma$ is 3-connected.

Proof. Indeed, from the above discussion, if $\Gamma$ is 3 connected, then $\Lambda\left(G_{\mathcal{P}}\right)$ is a gasket.

Conversely, if $\Gamma$ is not 2-connected, then $\Lambda\left(G_{\mathcal{P}}\right)$ is disconnected by Proposition 3.4, so it is not a gasket. On the other hand, if $\Gamma$ is 2-connected but not 3-connected, by Lemma 3.7 we have two vertices $v, w$ so that $v, w$ lie on the intersection of the boundaries of two faces $F_{1}$ and $F_{2}$. If $v, w$ are nonadjacent vertices in both $F_{1}$ and $F_{2}$, then the corresponding components $\Omega_{F_{1}}$ and $\Omega_{F_{2}}$ touch at two points corresponding to the two fixed points of the loxodromic element $g_{v} g_{w}$ (see $g_{C} g_{C^{\prime \prime}}$ in Figure 3.4). Thus, $\Lambda\left(G_{\mathcal{P}}\right)$ is not a gasket. If $v, w$ are nonadjacent vertices in $F_{1}$ but adjacent vertices in $F_{2}$, then $g_{v} g_{w}$ gives an accidental parabolic element. The corresponding component $\Omega_{F_{1}}$ is not a Jordan domain 
as the unique fixed point of the parabolic element $g_{v} g_{w}$ corresponds to two points on the ideal boundary of $\Omega_{F_{1}}$ (see $g_{C} g_{C^{\prime}}$ in Figure 3.4). Therefore, $\Lambda\left(G_{\mathcal{P}}\right)$ is not a gasket.

In the course of the proof, we have actually derived the following characterisation which is worth mentioning.

Proposition 3.11. Let $\mathcal{P}$ be a circle packing whose contact graph $\Gamma$ is not 3-connected and $G_{\mathcal{P}}$ be the associated kissing reflection group; then either

$\circ$ there exists a component of $\Omega\left(G_{\mathcal{P}}\right)$ which is not a Jordan domain; or

○ there exist two components of $\Omega\left(G_{\mathcal{P}}\right)$ whose closures touch at least at two points.

We are now able to prove the other direction of Proposition 3.6.

Lemma 3.12. If $\Gamma$ is 3-connected, then $G_{\mathcal{P}}$ is acylindrical.

Proof. Since $\Gamma$ is 3 -connected, it follows that the closures of any two different components of $\Omega\left(G_{\mathcal{P}}\right)$ intersect only at cusps. This means that there are no essential cylinder other than the pairing cylinders of the rank one cusps. So $G_{\mathcal{P}}$ is acylindrical.

Proof of Proposition 3.6. This follows from Lemma 3.8 and 3.12.

We remark that the unique configuration of pairs of circle packings given in Theorem 2.2 gives a kissing reflection group with totally geodesic boundary. This unique point in the deformation space of acylindrical manifolds is guaranteed by a theorem of McMullen [28].

\subsection{Deformation spaces of kissing reflection groups}

Throughout this section, we will use bold symbols, such as $\mathbf{G}, \mathbf{G}_{\Gamma}$, to represent the base point for the corresponding deformation spaces. We use regular symbols $G$ to represent the image of a representation in the deformation spaces. If the group is a kissing reflection group, we also use $G_{\mathcal{P}}$ if we want to emphasise the corresponding circle packing $\mathcal{P}$.

\section{Definition of $A H(\mathbf{G})$}

Let $\mathbf{G}$ be a finitely generated discrete subgroup of $\operatorname{Aut}^{ \pm}(\widehat{\mathbb{C}})$. A representation (that is, a group homomorphism) $\xi: \mathbf{G} \longrightarrow \operatorname{Aut}^{ \pm}(\widehat{\mathbb{C}})$ is said to be weakly type-preserving

1. if $\xi(g) \in \operatorname{Aut}^{+}(\widehat{\mathbb{C}})$ if and only if $g \in \operatorname{Aut}^{+}(\widehat{\mathbb{C}})$ and

2. if $g \in \operatorname{Aut}^{+}(\widehat{\mathbb{C}})$, then $\xi(g)$ is parabolic whenever $g$ is parabolic.

Note that a weakly type-preserving representation may send a loxodromic element to a parabolic one.

Definition 3.13. Let $\mathbf{G}$ be a finitely generated discrete subgroup of $\operatorname{Aut}^{ \pm}(\widehat{\mathbb{C}})$.

$$
\begin{aligned}
\mathrm{AH}(\mathbf{G}) & :=\{\xi: \mathbf{G} \longrightarrow G \text { is a weakly type-preserving isomorphism to } \\
& \text { a discrete subgroup } \left.G \text { of } \operatorname{Aut}^{ \pm}(\widehat{\mathbb{C}})\right\} / \sim,
\end{aligned}
$$

where $\xi_{1} \sim \xi_{2}$ if there exists a Möbius transformation $M$ such that

$$
\xi_{2}(g)=M \circ \xi_{1}(g) \circ M^{-1}, \text { for all } g \in \mathbf{G} .
$$

$\mathrm{AH}(\mathbf{G})$ inherits the quotient topology of algebraic convergence. Indeed, we say that a sequence of weakly type-preserving representations $\left\{\xi_{n}\right\}$ converges to $\xi$ algebraically if $\left\{\xi_{n}\left(g_{i}\right)\right\}$ converges to $\xi\left(g_{i}\right)$ as elements of $\mathrm{Aut}^{ \pm}(\widehat{\mathbb{C}})$ for (any) finite generating set $\left\{g_{i}\right\}$. 


\section{Quasiconformal deformation space of $\mathrm{G}$}

Recall that a Kleinian group is said to be geometrically finite if it has a finite-sided fundamental polyhedron. We say a finitely generated discrete subgroup of $\operatorname{Aut}^{ \pm}(\widehat{\mathbb{C}})$ is geometrically finite if the index 2 subgroup is geometrically finite.

Let $\mathbf{G}$ be a finitely generated, geometrically finite, discrete subgroup of $\operatorname{Aut}^{ \pm}(\widehat{\mathbb{C}})$. A group $G$ is called a quasiconformal deformation of $\mathbf{G}$ if there is a quasiconformal map $F: \widehat{\mathbb{C}} \longrightarrow \widehat{\mathbb{C}}$ that induces an isomorphism $\xi: \mathbf{G} \longrightarrow G$ such that $F \circ g(z)=\xi(g) \circ F(z)$ for all $g \in \mathbf{G}$ and $z \in \widehat{\mathbb{C}}$. Such a group $G$ is necessarily geometrically finite and discrete. The map $F$ is uniquely determined (up to normalisation) by its Beltrami differential on the domain of discontinuity $\Omega(\mathbf{G})$ (See [26, §5.1.2, Theorem 3.13.5]).

We define the quasiconformal deformation space of $\mathbf{G}$ as

$$
\mathcal{Q C}(\mathbf{G})=\{\xi \in \mathrm{AH}(\mathbf{G}): \xi \text { is induced by a quasiconformal deformation of } \mathbf{G}\} .
$$

By definition, $\mathcal{Q C}(\mathbf{G}) \subseteq \mathrm{AH}(\mathbf{G})$.

\section{Kissing reflection groups}

The above discussion is quite general and applies to any Kleinian (reflection) group. In the following, we will specialise to the case of a kissing reflection group.

Recall that different realisations of a fixed marked, connected simple plane graph $\Gamma$ as circle packings $\mathcal{P}$ give canonically isomorphic kissing reflection groups $G_{\mathcal{P}}$. Thus, the algebraic/quasiconformal deformation spaces of all such $G_{\mathcal{P}}$ can be canonically identified. However, in order to study these deformation spaces, it will be convenient to fix a (marked) circle packing realisation $\mathcal{P}$ of the (marked) graph $\Gamma$ and use the associated kissing reflection group

$$
\mathbf{G}_{\Gamma}:=G_{\mathcal{P}}
$$

as the base point. With this choice, we can and will use the notation $\mathrm{AH}(\Gamma)$ and $\mathcal{Q C}(\Gamma)$ to denote $\operatorname{AH}\left(\mathbf{G}_{\Gamma}\right)$ and $\mathcal{Q C}\left(\mathbf{G}_{\Gamma}\right)$, respectively.

Now let $\Gamma_{0}$ be a simple plane graph. The goal of this section is to describe the algebraic deformation space $\mathrm{AH}\left(\Gamma_{0}\right)$ and the closure $\overline{\mathcal{Q C}\left(\Gamma_{0}\right)}$ (of the quasiconformal deformation space) in $\mathrm{AH}\left(\Gamma_{0}\right)$.

In line with the convention introduced above, we choose a circle packing $\left\{\mathbf{C}_{1}, \ldots, \mathbf{C}_{n}\right\}$ realising $\Gamma_{0}$ and denote the reflection along $\mathbf{C}_{i}$ as $\rho_{i}$. Let $\mathbf{G}_{\Gamma_{0}}$ be the kissing reflection group generated by the reflections $\rho_{i}$. We remark that since we will be working with deformation spaces for several contact graphs, we use $\mathbf{G}_{\Gamma_{0}}$ to emphasise that the contact graph is $\Gamma_{0}$.

Let $G$ be a discrete subgroup of $\operatorname{Aut}^{ \pm}(\widehat{\mathbb{C}})$ and let $\xi: \mathbf{G}_{\Gamma_{0}} \longrightarrow G$ be a weakly type-preserving isomorphism. Since the union of the circles $\mathbf{C}_{1}, \ldots, \mathbf{C}_{n}$ is connected, for each $\rho_{i}$ there exists $\rho_{j}$ so that $\rho_{i} \circ \rho_{j}$ is parabolic. This implies that no $\xi\left(\rho_{i}\right)$ is the antipodal map. Hence, $\xi\left(\rho_{i}\right)$ is also a circular reflection. Assume that $\xi\left(\rho_{i}\right)$ is the reflection along some circle $C_{i}$. If $\rho_{i} \circ \rho_{j}$ is parabolic, then $\xi\left(\rho_{i}\right) \circ \xi\left(\rho_{j}\right)$ is also parabolic. Thus, $C_{i}$ is tangent to $C_{j}$ as well. This motivates the following definition.

Definition 3.14. Let $\Gamma$ be a simple plane graph with the same number of vertices as $\Gamma_{0}$. We say that $\Gamma$ abstractly dominates $\Gamma_{0}$, denoted by $\Gamma \geq_{a} \Gamma_{0}$, if there exists an embedding $\psi: \Gamma_{0} \longrightarrow \Gamma$ as abstract graphs (that is, if there exists a graph isomorphism between $\Gamma_{0}$ and a subgraph of $\Gamma$ ).

We say that $\Gamma$ dominates $\Gamma_{0}$, denoted by $\Gamma \geq \Gamma_{0}$, if there exists an embedding $\psi: \Gamma_{0} \longrightarrow \Gamma$ as plane graphs (that is, if there exists a graph isomorphism between $\Gamma_{0}$ and a subgraph of $\Gamma$ that extends to an orientation-preserving homeomorphism of $\widehat{\mathbb{C}}$ ).

We also define

$$
\operatorname{Emb}_{a}\left(\Gamma_{0}\right):=\left\{(\Gamma, \psi): \Gamma \geq_{a} \Gamma_{0} \text { and } \psi: \Gamma_{0} \longrightarrow \Gamma \text { is an embedding as abstract graphs }\right\}
$$



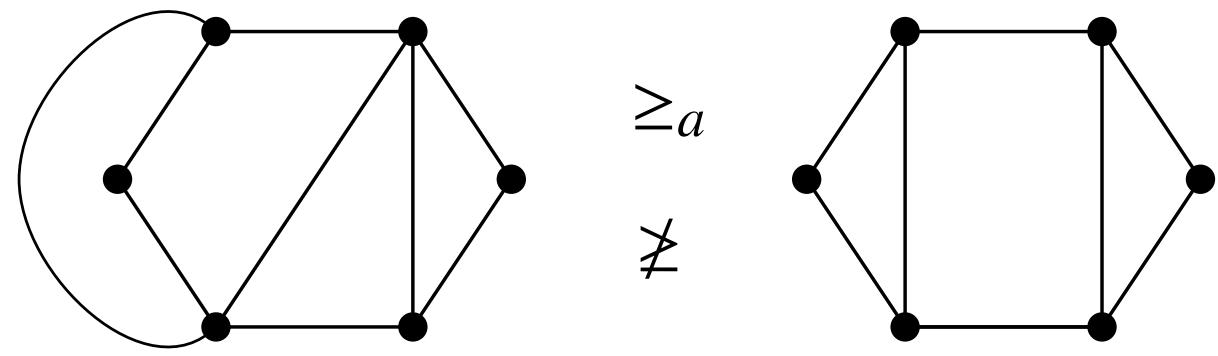

Figure 3.5. The graph on the left abstractly dominates the graph on the right, but no embedding of the right graph into the left graph respects the plane structure.

and

$$
\operatorname{Emb}_{p}\left(\Gamma_{0}\right):=\left\{(\Gamma, \psi): \Gamma \geq \Gamma_{0} \text { and } \psi: \Gamma_{0} \longrightarrow \Gamma \text { is an embedding as plane graphs }\right\}
$$

In other words, a simple plane graph $\Gamma$ abstractly dominates $\Gamma_{0}$ if $\Gamma$ (as an abstract graph) can be constructed from $\Gamma_{0}$ by introducing new edges, and it dominates $\Gamma_{0}$ if one can do this while respecting the plane structure.

We emphasise that the graph $\Gamma$ is always assumed to be planar, but the embedding $\psi$ may not respect the plane structure in $\operatorname{Emb}_{a}\left(\Gamma_{0}\right)$ (see Figure 3.5). We also remark that an element $(\Gamma, \psi)$ is identified with $\left(\Gamma^{\prime}, \psi^{\prime}\right)$ in $\operatorname{Emb}_{a}\left(\Gamma_{0}\right)$ or $\operatorname{Emb}_{p}\left(\Gamma_{0}\right)$ if $\psi^{\prime} \circ \psi^{-1}$ extends to an isomorphism between $\Gamma$ and $\Gamma^{\prime}$ as plane graphs.

We have the following lemma.

Lemma 3.15. Let $G$ be a discrete subgroup of $A u t^{ \pm}(\widehat{\mathbb{C}})$ and let $\xi: \mathbf{G}_{\Gamma_{0}} \longrightarrow G$ be a weakly typepreserving isomorphism. Then the simple plane graph $\Gamma$ associated with $G$ abstractly dominates $\Gamma_{0}$.

Conversely, if $\Gamma$ is a simple plane graph abstractly dominating $\Gamma_{0}$ and $G$ is a kissing reflection group associated with $\Gamma$, then there exists a weakly type-preserving isomorphism $\xi: \mathbf{G}_{\Gamma_{0}} \longrightarrow G$.

Proof. Let $G=\xi\left(\mathbf{G}_{\Gamma_{0}}\right)$ be a discrete faithful weakly type-preserving representation, then the reflections $\xi\left(\rho_{i}\right)$ along $C_{i}$ generate $G$. If there were a nontangential intersection between some $C_{i}$ and $C_{j}$, it would introduce a new relation between $\xi\left(\rho_{i}\right)$ and $\xi\left(\rho_{j}\right)$ by discreteness of $G$ (cf. [47, Chapter 5, Subsection 1.1]), which would contradict the assumption that $\xi$ is an isomorphism. Similarly, if there were circles $C_{i}, C_{j}, C_{k}$ touching at a point, then discreteness would give a new relation among $\xi\left(\rho_{i}\right), \xi\left(\rho_{j}\right)$ and $\xi\left(\rho_{k}\right)$, which would again lead to a contradiction. The above observations combined with the discussion preceding Definition 3.14 imply that $\left\{C_{1}, \ldots, C_{n}\right\}$ is a circle packing with associated contact graph $\Gamma$ abstractly dominating $\Gamma_{0}$.

Conversely, assume that $G$ is a kissing reflection group associated with a simple plane graph $\Gamma$ abstractly dominating $\Gamma_{0}$. Let $C_{i}$ be the circle corresponding to $\mathbf{C}_{i}$ under a particular embedding of $\Gamma_{0}$ into $\Gamma$ (as abstract graphs) and $g_{i}$ be the reflection along $C_{i}$. Defining $\xi: \mathbf{G}_{\Gamma_{0}} \longrightarrow G$ by $\xi\left(\rho_{i}\right)=g_{i}$, it is easy to check that $\xi$ is a weakly type-preserving isomorphism.

Note that for kissing reflection groups, the double of the polyhedron bounded by the half-planes associated to the circles $C_{i}$ is a fundamental polyhedron for the action of the index two Kleinian group on $\mathbb{H}^{3}$. Thus, we have the following corollary which is worth mentioning.

Corollary 3.16. Every group in $A H\left(\Gamma_{0}\right)$ is geometrically finite. 
Let $(\Gamma, \psi) \in \operatorname{Emb}_{p}\left(\Gamma_{0}\right)$ (respectively in $\operatorname{Emb}_{a}\left(\Gamma_{0}\right)$ ). Let us fix a circle packing $C_{1, \Gamma}, \ldots, C_{n, \Gamma}$ realising $\Gamma$, where $C_{i, \Gamma}$ corresponds to $\mathbf{C}_{i}$ under the embedding $\psi$ of $\Gamma_{0}$. Let $g_{i}$ be the associated reflection along $C_{i, \Gamma}$ and $\mathbf{G}_{\Gamma}=\left\langle g_{1}, \ldots, g_{n}\right\rangle$. Then Lemma 3.15 shows that

$$
\begin{aligned}
\xi_{(\Gamma, \psi)}: \mathbf{G}_{\Gamma_{0}} & \longrightarrow \mathbf{G}_{\Gamma} \\
\rho_{i} & \mapsto g_{i}
\end{aligned}
$$

is a weakly type-preserving isomorphism. Thus, $\mathcal{Q C}(\Gamma)=\mathcal{Q C}\left(\mathbf{G}_{\Gamma}\right)$ can be embedded in $\mathrm{AH}\left(\Gamma_{0}\right)=$ $\operatorname{AH}\left(\mathbf{G}_{\Gamma_{0}}\right)$. Indeed, if $\xi: \mathbf{G}_{\Gamma} \longrightarrow G$ represents an element in $\mathcal{Q C}(\Gamma)$, then

$$
\xi \circ \xi_{(\Gamma, \psi)}: \mathbf{G}_{\Gamma_{0}} \longrightarrow G
$$

is a weakly type-preserving isomorphism. It can be checked that the map $\xi \mapsto \xi \circ \xi_{(\Gamma, \psi)}$ gives an embedding of $\mathcal{Q C}(\Gamma)$ into $\mathrm{AH}\left(\Gamma_{0}\right)$. We shall identify the space $\mathcal{Q C}(\Gamma)$ with its image in $\mathrm{AH}\left(\Gamma_{0}\right)$ under this embedding.

\section{Proposition 3.17.}

$$
A H\left(\Gamma_{0}\right)=\bigcup_{(\Gamma, \psi) \in \operatorname{Emb}_{a}\left(\Gamma_{0}\right)} \mathcal{Q C}(\Gamma)
$$

and

$$
\overline{\mathcal{Q C}\left(\Gamma_{0}\right)}=\bigcup_{(\Gamma, \psi) \in \operatorname{Emb}_{p}\left(\Gamma_{0}\right)} \mathcal{Q C}(\Gamma) .
$$

The proof of this proposition will be furnished after a discussion of pinching deformations. Once the connection with pinching deformation is established, the result can be derived from [35].

\section{The perspective of pinching deformation}

For the general discussion of pinching deformations, let us fix an arbitrary 2-connected simple plane graph $\Gamma$, a circle packing $\mathcal{P}$ with contact graph isomorphic to $\Gamma$ and the associated kissing reflection group $\mathbf{G}=G_{\mathcal{P}}$, which we think of as the base point of the quasiconformal deformation space $\mathcal{Q C}(\Gamma)$. Since $\Gamma$ is the only graph appearing in this discussion (until the proof Proposition 3.17), we omit the subscript ' $\Gamma$ ' from the base point $\mathbf{G}$.

Let $F$ be a face of $\Gamma$ and $R_{F}$ be the associated component of $\partial \mathcal{M}(\mathbf{G})$. Let $C \in \mathcal{P}$ be a circle on the boundary of $\Pi_{F}$. Then the reflection along $C$ descends to an anti-conformal involution on $R_{F}$, which we shall denote as

$$
\sigma_{F}: R_{F} \longrightarrow R_{F}
$$

Note that different choices of the circle descend to the same involution. It is known that for boundary incompressible geometrically finite Kleinian groups, the quasiconformal deformation space is the product of the Teichmüller spaces of the components of the conformal boundary (see [26, Theorem 5.1.3]):

$$
\mathcal{Q C}(\widetilde{\mathbf{G}})=\prod_{F \text { face of } \Gamma} \operatorname{Teich}\left(R_{F}\right),
$$

where $\widetilde{\mathbf{G}}$ is the index two subgroup of $\mathbf{G}$ consisting of orientation-preserving elements. 
We denote by $\operatorname{Teich}^{\sigma_{F}}\left(R_{F}\right) \subseteq \operatorname{Teich}\left(R_{F}\right)$ those elements corresponding to $\sigma_{F}$-invariant quasiconformal deformations of $R_{F}$. Then

$$
\mathcal{Q C}(\mathbf{G})=\prod_{F \text { face of } \Gamma} \operatorname{Teich}^{\sigma_{F}}\left(R_{F}\right)
$$

Indeed, any element in $\prod_{F}$ face of $\Gamma$ Teich ${ }^{\sigma_{F}}\left(R_{F}\right)$ is uniquely determined by the associated Beltrami differential on $\Pi_{F}$, which can be pulled back by $\mathbf{G}$ to produce a $\mathbf{G}$-invariant Beltrami differential on $\widehat{\mathbb{C}}$. Such a Beltrami differential can be uniformised by the measurable Riemann mapping theorem.

Recall that $\Pi_{F}$ is an ideal polygon. Note that the component of $\Omega(\mathbf{G})$ containing $\Pi_{F}$ is simply connected and hence $\Pi_{F}$ inherits the hyperbolic metric from the corresponding component of $\Omega(\mathbf{G})$. Then $R_{F}$ is simply the double of $\Pi_{F}$. We claim that the only $\sigma_{F}$-invariant geodesics are those simple closed curves $\widetilde{\gamma}_{v w}^{F}$ on $R_{F}$ (see the discussion following Proposition 3.6 for the definition of $\widetilde{\gamma}_{v w}^{F}$ ), where $v, w$ are two nonadjacent vertices on the boundary of $F$. Indeed, any $\sigma_{F}$-invariant geodesic would intersect the boundary of the ideal polygon perpendicularly and the $\widetilde{\gamma}_{v w}^{F}$ are the only geodesics satisfying this property. We denote the associated geodesic arc in $\Pi_{F}$ by $\gamma_{v w}^{F}$.

We define a multicurve on a surface as a disjoint union of simple closed curves, such that no two components are homotopic. It is said to be weighted if a nonnegative number is assigned to each component. We shall identify two multicurves if they are homotopic to each other.

Let $\mathcal{T}$ be a triangulation of $F$ obtained by adding new edges connecting the vertices of $F$. Since each additional edge in this triangulation connects two nonadjacent vertices of $F$, we can associate a multicurve $\widetilde{\alpha}_{\mathcal{T}}^{F}$ on $R_{F}$ consisting of all $\widetilde{\gamma}_{v w}^{F}$, where $v w$ is a new edge in $\mathcal{T}$. A marking of the graph $\Gamma$ also gives a marking of the multicurve. We use $\alpha_{\mathcal{T}}^{F}$ to denote the multi-arc in the hyperbolic ideal polygon $\Pi_{F}$. Since $\mathcal{T}$ is a triangulation, the multicurve $\widetilde{\alpha}_{\mathcal{T}}^{F}$ yields a pants decomposition of the punctured sphere $R_{F}$ such that the boundary components of every pair of pants are punctures or simple closed curves in $\widetilde{\alpha}_{\mathcal{T}}^{F}$. Thus, the complex structures of these pairs of pants are uniquely determined by the lengths of the marked multicurve $\widetilde{\alpha}_{\mathcal{T}}^{F}$ (cf. [15, Theorem 3.5]). Since $R_{F}$ is invariant under the anti-conformal involution $\sigma_{F}$, the surface $R_{F}$ is uniquely determined by the complex structures of the above pairs of pants and hence by the lengths of the marked multicurve $\widetilde{\alpha}_{\mathcal{T}}^{F}$. If $F$ has $m$ sides, then any triangulation of $F$ has $m-3$ additional edges. Thus, we have the analogue of Fenchel-Nielsen coordinates on

$$
\operatorname{Teich}^{\sigma_{F}}\left(R_{F}\right)=\mathbb{R}_{+}^{m-3}
$$

by assigning to each element of Teich ${ }^{\sigma_{F}}\left(R_{F}\right)$ the lengths of the marked multicurve $\widetilde{\alpha}_{\mathcal{T}}^{F}$ (or, equivalently, the lengths of the marked multi-arc $\alpha_{\mathcal{T}}^{F}$ ). Note that with these lengths, the multi-arcs and the multicurves become weighted. Note that different triangulations yield different coordinates. We also remark that unlike classical Fenchel-Nielsen coordinates on Teichmüller spaces, Fenchel-Nielsen coordinates on Teich $^{\sigma_{F}}\left(R_{F}\right)$ do not contain twist parameters precisely due to the $\sigma_{F}$-invariance of quasiconformal deformations (cf. [15, §3.2]).

In order to degenerate in Teich ${ }^{\sigma_{F}}\left(R_{F}\right)$, the length of some arc $\gamma_{v w}^{F}$ must shrink to 0 . The above discussion implies that the closure of Teich ${ }^{\sigma_{F}}\left(R_{F}\right)$ in the Thurston compactification consists only of weighted $\sigma_{F}$-invariant multicurves $\widetilde{\alpha}^{F}$. If $S_{k} \in$ Teich $^{\sigma_{F}}\left(R_{F}\right)$ converges to a weighted $\sigma_{F}$-invariant multicurve $\widetilde{\alpha}^{F}$, then we say that $S_{k}$ is a pinching deformation on $\widetilde{\alpha}^{F}$. More generally, we say that $G_{k} \in \mathcal{Q C}(\mathbf{G})$ is a pinching deformation on $\widetilde{\alpha}=\bigcup \widetilde{\alpha}^{F}$ if for each face $F$, the conformal boundary associated to $F$ is a pinching deformation on $\widetilde{\alpha}^{F}$.

Given two curves $\widetilde{\gamma}_{v w}^{F} \subseteq R_{F}$ and $\widetilde{\gamma}_{u t}^{F^{\prime}} \subseteq R_{F^{\prime}}$, we say that they are parallel if they are homotopic in the 3-manifold $\mathcal{M}\left(G_{\mathcal{P}}\right)$. Since the curve $\widetilde{\gamma}_{v w}^{F}$ corresponds to the element $g_{v} g_{w}$, we get that $\widetilde{\gamma}_{v w}^{F}$ and $\tilde{\gamma}_{u t}^{F^{\prime}}$ are parallel if and only if (possibly after switching the order) $v=u$ and $w=t$. Note that in this case, $g_{v} g_{w} \in \operatorname{stab}\left(\Omega_{F}\right) \cap \operatorname{stab}\left(\Omega_{F^{\prime}}\right)$ and $v, w$ are on the common boundaries of $F$ and $F^{\prime}$. (See, for example, Figure 3.4 where $v, w$ correspond to the circles $C, C^{\prime \prime}$, where the curve $\tilde{\gamma}_{v w}^{F_{1}}$ is parallel with $\tilde{\gamma}_{v w}^{F_{2}}$.) 
Let $\widetilde{\alpha}=\bigcup \widetilde{\alpha}^{F}$ be a union of $\sigma_{F}$-invariant multicurves. We say that $\widetilde{\alpha}$ is a nonparallel multicurve if no two components are parallel. We note that nonparallel multicurves $\widetilde{\alpha}$ for $\mathbf{G}$ are in one-to-one correspondence with the simple plane graphs that dominate $\Gamma$. Indeed, any multicurve $\widetilde{\alpha}=\bigcup \widetilde{\alpha}^{F}$ corresponds to a plane graph that dominates $\Gamma$. The nonparallel condition is equivalent to the condition that the graph is simple.

We now apply the general discussion carried out above to prove Proposition 3.17.

Proof of Proposition 3.17. The first equality follows from Lemma 3.15.

We first show $\bigcup_{(\Gamma, \psi) \in \operatorname{Emb}_{p}\left(\Gamma_{0}\right)} \mathcal{Q C}(\Gamma) \subseteq \overline{\mathcal{Q C}\left(\Gamma_{0}\right)}$. Let $\Gamma$ be a simple plane graph that dominates $\Gamma_{0}$ with embedding $\psi: \Gamma_{0} \longrightarrow \Gamma$ as plane graphs. It follows from the discussion on pinching deformations that there exists a nonparallel multicurve $\widetilde{\alpha} \subseteq \partial \mathcal{M}\left(\mathbf{G}_{\Gamma_{0}}\right)$ associated to $\Gamma$. We complete $\Gamma$ to a triangulation $\mathcal{T}$ by adding edges. As before, $\mathcal{T}$ gives a $\sigma$-invariant multicurve $\widetilde{\beta} \subseteq \partial \mathcal{M}\left(\mathbf{G}_{\Gamma_{0}}\right)$ which contains $\widetilde{\alpha}$. We set $\widetilde{\beta}=\widetilde{\alpha} \sqcup \widetilde{\alpha}^{\prime}$. Then the lengths of the multicurve $\widetilde{\alpha}^{\prime}$ give a parametrisation of $\mathcal{Q C}(\Gamma)$ and the lengths of the multicurve $\widetilde{\beta}$ give a parametrisation of $\mathcal{Q C}\left(\Gamma_{0}\right)$. Now given any element $\vec{l}=\left(l_{\gamma}: \gamma \in \widetilde{\alpha}^{\prime}\right) \in \mathcal{Q C}(\Gamma)$, we show that it can be realised as the algebraic limit of a sequence in $\mathcal{Q C}\left(\Gamma_{0}\right)$.

Such a construction is standard and follows directly from [35, Theorem 5.1] (see also [27]). For completeness, we sketch the proof here. Let $G \in \mathcal{Q C}\left(\Gamma_{0}\right)$ be so that the length of the multicurve $\widetilde{\alpha}^{\prime}$ is $\vec{l}$. We assume that $\widetilde{\beta}$ is realised by hyperbolic geodesics in $\partial \mathcal{M}(G)$. Let $A \subseteq \partial \mathcal{M}(G)$ be an $\epsilon$-neighbourhood of $\widetilde{\alpha}$. We choose $\epsilon$ small enough so that $A$ is disjoint from $\widetilde{\alpha}^{\prime}$ and each component contains only one component of $\widetilde{\alpha}$. We construct a sequence of quasiconformal deformations supported on $A$ so that the modulus of each annulus in $A$ tends to infinity while $\widetilde{\alpha}$ remains as core curves. Since the multicurve $\widetilde{\alpha}$ is nonparallel, Thurston's hyperbolisation theorem guarantees a convergent subsequence (see [35] for more details). This algebraic limit is a kissing reflection group. By construction, the contact graph of such a limiting kissing reflection group is $\Gamma$ and the lengths of $\widetilde{\alpha}^{\prime}$ is $\vec{l}$.

Conversely, if a sequence of quasiconformal deformations $\xi_{n}: \mathbf{G}_{\Gamma_{0}} \longrightarrow G_{n}$ of $\mathbf{G}_{\Gamma_{0}}$ converges to $\xi_{\infty}: \mathbf{G}_{\Gamma_{0}} \longrightarrow G$ algebraically, then $G$ is a kissing reflection group. Since $\left\{\xi_{n}\left(g_{i}\right)\right\}$ converges, where $g_{i}$ are the standard generators, the contact graph for $G$ dominates $\Gamma_{0}$. The embedding $\psi: \Gamma_{0} \longrightarrow \Gamma$ (respecting the plane structure) comes from the identification of the generators.

\subsection{Quasi-Fuchsian space and mating locus}

The above discussion applies to the special case of quasi-Fuchsian space. This space is related to the mating locus for critically fixed anti-rational maps.

Let $\Gamma_{d}$ be the marked $d+1$-sided polygonal graph; that is, $\Gamma_{d}$ contains $d+1$ vertices $v_{1}, \ldots, v_{d+1}$ with edges $v_{i} v_{i+1}$ where indices are understood modulo $d+1$. We choose the most symmetric circle packing $\mathcal{P}_{d}$ realising $\Gamma_{d}$. More precisely, consider the ideal $(d+1)$-gon in $\mathbb{D} \cong \mathbb{H}^{2}$ with vertices at the $(d+1)$-st roots of unity. The edges of this ideal $(d+1)$-gon are arcs of $d+1$ circles and we label these circles as $\mathcal{P}_{d}:=\left\{\mathbf{C}_{1}, \ldots, \mathbf{C}_{d+1}\right\}$, where we index them counterclockwise such that $\mathbf{C}_{1}$ passes through 1 and $e^{2 \pi i /(d+1)}$. Let $\mathbf{G}_{d}$ be the kissing reflection group associated to $\mathcal{P}_{d}$. Note that $\widetilde{\mathbf{G}}_{d}$ is a Fuchsian group. We remark that since any embedding of the polygonal graph $\Gamma_{d}$ into a graph $\Gamma$ abstractly dominating $\Gamma_{d}$ respects the plane structure, we have that $\mathrm{AH}\left(\Gamma_{d}\right)=\overline{\mathcal{Q C}\left(\Gamma_{d}\right)}$.

Recall that a graph $\Gamma$ is said to be Hamiltonian if there exists a cycle which passes through each vertex exactly once. Since a simple plane graph with $d+1$ vertices is Hamiltonian if and only if it dominates $\Gamma_{d}$, the following proposition follows immediately from Proposition 3.17.

Proposition 3.18. Let $\Gamma$ be a simple plane graph with $d+1$ vertices; then any kissing reflection group $G$ with contact graph $\Gamma$ is in the closure $\overline{\mathcal{Q C}\left(\Gamma_{d}\right)}$ if and only if $\Gamma$ is Hamiltonian.

Let $\Gamma$ be a marked Hamiltonian simple plane graph with a Hamiltonian cycle $C=\left(v_{1}, \ldots, v_{d+1}\right)$. Let $G:=\left\langle g_{1}, \ldots, g_{d+1}\right\rangle$ be a kissing reflection group with contact graph $\Gamma$. The Hamiltonian cycle divides the fundamental domain $\Pi \subseteq \widehat{\mathbb{C}}$ into two parts and we denote them as $\Pi^{+}$and $\Pi^{-}$, where we assume that the Hamiltonian cycle is positively oriented on the boundary of $\Pi^{+}$and negatively oriented on the 
boundary of $\Pi^{-}$(see Figure 3.4). We denote

$$
\Omega^{ \pm}:=\bigcup_{g \in G} g \cdot \Pi^{ \pm}
$$

Since each $\Omega^{ \pm}$is $G$-invariant, we have $\Lambda(G)=\partial \Omega^{+}=\partial \Omega^{-}$.

As in Subsection 3.1, set

$$
\Pi^{1, \pm}=\bigcup_{i=1}^{d+1} g_{i} \cdot \Pi^{ \pm} \text {, and } \Pi^{j+1, \pm}=\bigcup_{i=1}^{d+1} g_{i} \cdot\left(\Pi^{j, \pm} \backslash \bar{D}_{i}\right) .
$$

For consistency, we also set $\Pi^{0, \pm}=\Pi^{ \pm}$. Then the arguments of the proof of Lemma 3.2 show that

$$
\Pi^{l, \pm}=\bigcup_{|g|=l} g \cdot \Pi^{ \pm} .
$$

Lemma 3.19. The closures $\overline{\Omega^{+}}$and $\overline{\Omega^{-}}$are connected.

Proof. We shall prove by induction that the closures $\overline{\bigcup_{i=0}^{n} \Pi^{i,+}}$ and $\overline{\bigcup_{i=0}^{n} \Pi^{i,+} \backslash \bar{D}_{j}}$ are connected for all $n$ and $j$.

Indeed, the base case is true as $\overline{\Pi^{+}}$and $\overline{\Pi^{+} \backslash \bar{D}_{j}}$ are connected for all $j$. Assume that $\overline{\bigcup_{i=0}^{n} \Pi^{i,+}}$ and $\overline{\bigcup_{i=0}^{n} \Pi^{i,+} \backslash \bar{D}_{j}}$ are connected for all $j$. Note that

$$
\overline{\bigcup_{i=0}^{n+1} \Pi^{i,+}}=\overline{\Pi^{+} \cup \bigcup_{j=1}^{d+1} g_{j} \cdot\left(\bigcup_{i=0}^{n} \Pi^{i,+} \backslash \bar{D}_{j}\right)} .
$$

By the induction hypothesis, $\overline{g_{j} \cdot\left(\bigcup_{i=0}^{n} \Pi^{i,+} \backslash \bar{D}_{j}\right)}$ is connected. Since each

$$
\overline{g_{j} \cdot\left(\bigcup_{i=0}^{n} \Pi^{i,+} \backslash \bar{D}_{j}\right)}
$$

intersects $\overline{\Pi^{+}}$along an arc of $C_{j}=\partial D_{j}$, we have that $\overline{\bigcup_{i=0}^{n+1} \Pi^{i,+}}$ is also connected.

Similarly,

$$
\overline{\bigcup_{i=0}^{n+1} \Pi^{i,+} \backslash \bar{D}_{j}}=\overline{\Pi^{+} \cup \bigcup_{k \neq j} g_{k} \cdot\left(\bigcup_{i=0}^{n} \Pi^{i,+} \backslash \bar{D}_{k}\right)}
$$

is connected for any $j$.

Connectedness of $\overline{\Omega^{-}}$is proved in the same way.

\section{Matings of function kissing reflection groups}

We say that a kissing reflection group $G$ with connected limit set is a function kissing reflection group if there is a component $\Omega_{0}$ of $\Omega(G)$ invariant under $G$. This terminology was traditionally used in complex analysis as one can construct Poincaré series, differentials and functions on it.

We say that a simple plane graph $\Gamma$ with $n$ vertices is outerplanar if it has a face with all $n$ vertices on its boundary. We also call this face the outer face. Note that a 2-connected outerplanar graph is Hamiltonian with a unique Hamiltonian cycle. The following proposition characterises function kissing reflection groups in terms of their contact graphs. 


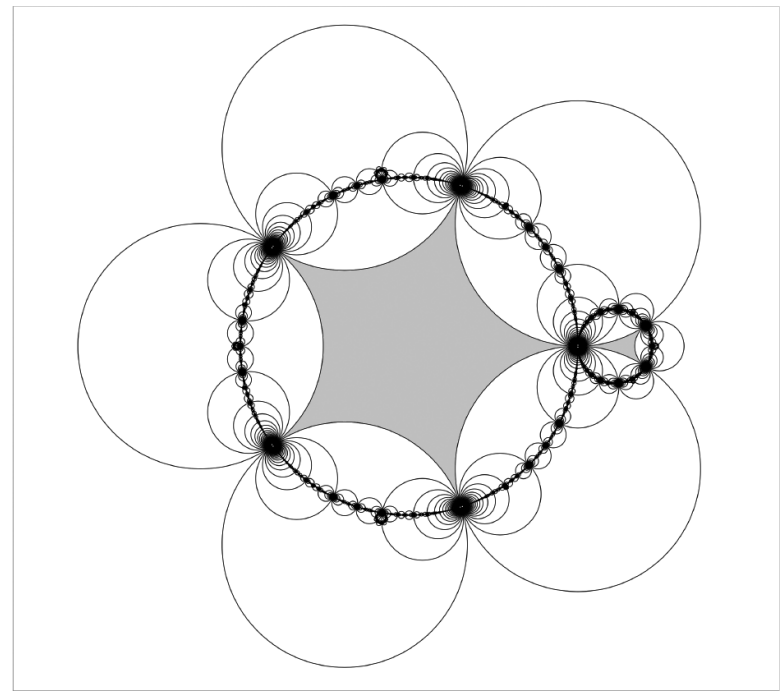

Figure 3.6. The limit set of a function kissing reflection group $H$ with an outerplanar contact graph. The grey region represents the part $\Pi_{b}$ of the fundamental domain $\Pi(H)$ corresponding to the nonouter faces of $\Gamma(H)$. The kissing reflection group $G$ shown in Figure 3.4 can be constructed by pinching a simple closed curve for $H$.

Proposition 3.20. A kissing reflection group $G$ is a function group if and only if its contact graph $\Gamma$ is 2-connected and outerplanar.

Proof. If $\Gamma$ is outerplanar, then let $\Omega_{F}$ be the component of $\Omega(G)$ associated to the outer face $F$. It is easy to see that the standard generating set fixes $\Omega_{F}$, so $\Omega_{F}$ is invariant under $G$.

Conversely, assume that $\Gamma$ is not outerplanar. Since $\Pi$ is a fundamental domain of the action of $G$ on $\Omega(G)$, any $G$-invariant component of $\Omega(G)$ must correspond to some face of $\Gamma$. Let $F$ be a face of $\Gamma$. Since $\Gamma$ is not outerplanar, there exists a vertex $v$ which is not on the boundary of $F$. Thus, $g_{v} \cdot \Pi_{F}$ is not in $\Omega_{F}$ and hence $\Omega_{F}$ is not invariant under $G$.

For a function kissing reflection group $G$, we set $\Omega_{b}:=\Omega(G) \backslash \Omega_{0}$, where $\Omega_{0}$ is $G$-invariant. Similarly, we shall use the notation $\Pi_{b}:=\Pi \cap \Omega_{b}$ (see Figure 3.6) and $\Pi_{b}^{i}:=\Pi^{i} \cap \Omega_{b}$, for $i \geq 0$ (see Lemma 3.2). We call the closure $\overline{\Omega_{b}}=\widehat{\mathbb{C}} \backslash \Omega_{0}$ the filled limit set for the function kissing reflection group $G$ and denote it by $\mathcal{K}(G)$.

Let $G^{ \pm}$be two function kissing reflection groups with the same number of vertices in their contact graphs. We say that a kissing reflection group $G$ is a geometric mating of $G^{+}$and $G^{-}$if we have

○ a decomposition $\Omega(G)=\Omega^{+}(G) \sqcup \Omega^{-}(G)$ with $\Lambda(G)=\partial \Omega^{+}(G)=\partial \Omega^{-}(G)$;

$\circ$ weakly type-preserving isomorphisms $\phi^{ \pm}: G^{ \pm} \longrightarrow G$;

○ continuous surjections $\psi^{ \pm}: \mathcal{K}\left(G^{ \pm}\right) \longrightarrow \overline{\Omega^{ \pm}(G)}$ which are conformal between the interior $\mathcal{K}\left(G^{ \pm}\right)$ and $\Omega^{ \pm}(G)$ such that for any $g \in G^{ \pm},\left.\psi^{ \pm} \circ g\right|_{\mathcal{K}\left(G^{ \pm}\right)}=\phi^{ \pm}(g) \circ \psi^{ \pm}$.

Note that by the semi-conjugacy relation, the sets $\Omega^{ \pm}(G)$ are $G$-invariant. We shall now complete the proof of the group part of Theorem 1.2.

Proposition 3.21. A kissing reflection group $G$ is a geometric mating of two function kissing reflection groups if and only if the contact graph $\Gamma(G)$ of $G$ is Hamiltonian.

Proof. If $G$ is a geometric mating of $G^{ \pm}$, then we have a decomposition $\Omega(G)=\Omega^{+}(G) \sqcup \Omega^{-}(G)$ with $\Lambda(G)=\partial \Omega^{+}(G)=\partial \Omega^{-}(G)$. This gives a decomposition of $\Pi(G)=\Pi^{+} \sqcup \Pi^{-}$and thus a decomposition of the contact graph $\Gamma(G)=\Gamma^{+} \cup \Gamma^{-}$. More precisely, the vertex sets of $\Gamma^{ \pm}$coincide with 
that of $\Gamma(G)$ and there is an edge in $\Gamma^{ \pm}$connecting two vertices if and only if the point of intersection of the corresponding two circles lies in $\partial \Pi^{ \pm}$. Since the action of $G^{ \pm}$on $\mathcal{K}\left(G^{ \pm}\right)$is semi-conjugate to $G$ on $\overline{\Omega^{ \pm}(G)}$, it follows that $\Gamma^{ \pm}$is isomorphic to $\Gamma\left(G^{ \pm}\right)$as plane graphs. Thus, the intersection of $\Gamma^{+}$ and $\Gamma^{-}$(which is the common boundary of the outer faces of $\Gamma^{+}$and $\Gamma^{-}$) gives a Hamiltonian cycle for $\Gamma(G)$.

Conversely, if $\Gamma(G)$ is Hamiltonian, then a Hamiltonian cycle yields a decomposition $\Gamma(G)=\Gamma^{+} \cup \Gamma^{-}$, where $\Gamma^{+}$and $\Gamma^{-}$are outerplanar graphs with vertex sets equal to that of $\Gamma(G)$. We construct function kissing reflection groups $G^{ \pm}$with contact graphs $\Gamma\left(G^{ \pm}\right)=\Gamma^{ \pm}$. Note that we have a natural embedding of $\Gamma\left(G^{ \pm}\right)$into $\Gamma(G)$ (as plane graphs), which gives an identification of vertices and non-outer faces. Thus, we have weakly type-preserving isomorphisms $\phi^{ \pm}: G^{ \pm} \longrightarrow G$ coming from the identification of vertices and Proposition 3.17. By quasiconformal deformation, we may assume that for each non-outer face $F \in \Gamma\left(G^{ \pm}\right)$, the associated conformal boundary component $R_{F}\left(G^{ \pm}\right) \subseteq \partial \mathcal{M}\left(G^{ \pm}\right)$is conformally equivalent to the corresponding conformal boundary component $R_{F}(G) \subseteq \partial \mathcal{M}(G)$.

The existence of the desired continuous map $\psi^{+}$can be derived from a more general statement on the existence of Cannon-Thurston maps (see [32, Theorem 4.2]). For completeness and making the proof self-contained, we give an explicit construction of $\psi^{+}: \mathcal{K}\left(G^{+}\right) \longrightarrow \overline{\Omega^{+}(G)}$. This construction is done in levels: we start with a homeomorphism

$$
\psi_{0}^{+}: \overline{\Pi_{b}^{0}\left(G^{+}\right)} \longrightarrow \overline{\Pi^{0++}}
$$

This can be chosen to be conformal on the interior as the associated conformal boundaries are assumed to be conformally equivalent. Assuming that

$$
\psi_{i}^{+}: \overline{\bigcup_{j=0}^{i} \Pi_{b}^{j}\left(G^{+}\right)} \longrightarrow \overline{\bigcup_{j=0}^{i} \Pi^{j,+}}
$$

is constructed, we extend $\psi_{i}^{+}$to $\psi_{i+1}^{+}$by setting

$$
\psi_{i+1}^{+}(z):=\phi^{+}\left(g_{k}\right) \circ \psi_{i}^{+} \circ g_{k}(z)
$$

if $z \in \Pi_{b}^{i+1}\left(G^{+}\right) \cap D_{k}$. It is easy to check by induction that $\psi_{i+1}^{+}$is a homeomorphism which is conformal on the interior (see Figure 3.4 and Figure 3.6).

Let $P^{0}\left(G^{+}\right):=\overline{\Pi_{b}^{0}\left(G^{+}\right)} \backslash \Pi_{b}^{0}\left(G^{+}\right)$; then $P^{0}\left(G^{+}\right)$consists of cusps where various components of $\Pi_{b}^{0}\left(G^{+}\right)$touch. Let $P^{\infty}\left(G^{+}\right):=\bigcup_{g \in G^{+}} g \cdot P^{0}\left(G^{+}\right)$. Then $P^{\infty}\left(G^{+}\right)$is dense in $\Lambda\left(G^{+}\right)$. We define $P^{\infty,+}$ similarly (where, $P^{0,+}:=\overline{\Pi^{0,+}} \backslash \Pi^{0,+}$ ) and note that $P^{\infty,+}$ is dense in $\partial \Omega^{+}(G)=\Lambda(G)$.

Note that $\psi_{i}^{+}=\psi_{j}^{+}$on $P^{i}\left(G^{+}\right)$for all $j \geq i$. Thus, we have a well-defined limit $\psi^{+}: P^{\infty}\left(G^{+}\right) \longrightarrow P^{\infty,+}$. We claim that $\psi^{+}$is uniformly continuous on $P^{\infty}\left(G^{+}\right)$. For an arbitrary $\epsilon>0$, there exists $N$ such that all disks in $\mathcal{D}^{N}(G)$ have spherical diameter $<\epsilon$ by Lemma 3.3. Choose $\delta$ so that any two nonadjacent disks in $\mathcal{D}^{N}\left(G^{+}\right)$are separated in spherical metric by $\delta$. Then if $x, y \in P^{\infty}\left(G^{+}\right)$with $d(x, y)<\delta$, they must lie in two adjacent disks of $\mathcal{D}^{N}\left(G^{+}\right)$. Thus, $\psi_{i}^{+}(x)$ and $\psi_{i}^{+}(y)$ (whenever defined) lie in two adjacent disks of $\mathcal{D}^{N}(G)$ for all $i \geq N$. Hence, $d\left(\psi_{i}^{+}(x), \psi_{i}^{+}(y)\right)<2 \epsilon$ for all $i \geq N$. This shows that $\psi^{+}$ is uniformly continuous on $P^{\infty}\left(G^{+}\right)$.

Thus, we have a continuous extension $\psi^{+}: \mathcal{K}\left(G^{+}\right) \longrightarrow \overline{\Omega^{+}(G)}$. It is easy to check that $\psi^{+}$is surjective, conformal on the interior and equivariant with respect to the actions of $G^{+}$and $G$.

The same proof gives the construction for $\psi^{-}$. This shows that $G$ is a geometric mating of $G^{+}$ and $G^{-}$.

Note that from the proof of Proposition 3.21, we see that each (marked) Hamiltonian cycle $H$ gives a decomposition of the graph $\Gamma=\Gamma^{+} \cup \Gamma^{-}$and hence an unmating of a kissing reflection group. From the pinching perspective as in the proof of Proposition 3.17, this (marked) Hamiltonian cycle 
also gives a pair of nonparallel multicurves. This pair of multicurves can be constructed explicitly from the decomposition of $\Gamma$. Indeed, each edge in $\Gamma^{ \pm}-H$ gives a pair of nonadjacent vertices in $H$. The corresponding $\sigma$-invariant curve comes from these nonadjacent vertices in $H$. If a marking is not specified on $H$, this pair of multicurves is defined only up to simultaneous change of coordinates on the two conformal boundaries.

\subsection{Nielsen maps for kissing reflection groups}

Let $\Gamma$ be a simple plane graph and $G_{\Gamma}$ be a kissing reflection group with contact graph $\Gamma$. Recall that $\overline{\mathcal{D}}=\bigcup_{j=1}^{n} \overline{D_{j}}$ is defined as the union of the closure of the disks for the associated circle packing. We define the Nielsen map $\mathcal{N}_{\Gamma}: \overline{\mathcal{D}} \longrightarrow \widehat{\mathbb{C}}$ by

$$
\mathcal{N}_{\Gamma}(z)=g_{j}(z) \text { if } z \in \overline{D_{j}}
$$

Let us now assume that $\Gamma$ is 2 -connected; then the limit set $\Lambda\left(G_{\Gamma}\right)$ is connected. Thus, each component of $\Omega\left(G_{\Gamma}\right)$ is a topological disk. Let $F$ be a face with $d+1$ sides of $\Gamma$ and let $\Omega_{F}$ be the component of $\Omega(G)$ containing $\Pi_{F}$. By a quasiconformal deformation, we can assume that the restriction of $G_{\Gamma}$ on $\Omega_{F}$ is conformally conjugate to the regular ideal $d+1$-gon reflection group on $\mathbb{D} \cong \mathbb{H}^{2}$.

For the regular ideal $d+1$-gon reflection group $\mathbf{G}_{d}$ (whose associated contact graph is $\Gamma_{d}$ ), the $d+1$ disks yield a Markov partition for the action of the Nielsen map $\mathcal{N}_{d} \equiv \mathcal{N}_{\Gamma_{d}}$ on the limit set $\Lambda\left(\mathbf{G}_{d}\right)=\mathbb{S}^{1}$. The diameters of the preimages of these disks under $\mathcal{N}_{d}$ shrink to 0 uniformly by Lemma 3.3 and hence the Nielsen map $\mathcal{N}_{d}$ is topologically conjugate to $z \mapsto \bar{z}^{d}$ on $\mathbb{S}^{1}$ (cf. [24, §4]).

Therefore, the Nielsen map $\mathcal{N}_{\Gamma}$ is topologically conjugate to $\bar{z}^{d}$ on the ideal boundary of $\Omega_{F}$. This allows us to replace the dynamics of $\mathcal{N}_{\Gamma}$ on $\Omega_{F}$ by $\bar{z}^{d}$ for every face of $\Gamma$ and obtain a globally defined orientation-reversing branched covering $\mathcal{G}_{\Gamma}: \widehat{\mathbb{C}} \longrightarrow \widehat{\mathbb{C}}$.

More precisely, let $\phi: \mathbb{D} \longrightarrow \Omega_{F}$ be a conformal conjugacy between $\mathcal{N}_{d}$ and $\mathcal{N}_{\Gamma}$, which extends to a topological conjugacy from the ideal boundary $\mathbb{S}^{1}=I(\mathbb{D})=\partial \mathbb{D}$ onto $I\left(\Omega_{F}\right)$. Let $\psi: \overline{\mathbb{D}} \longrightarrow \overline{\mathbb{D}}$ be an arbitrary homeomorphic extension of the topological conjugacy between $\left.\bar{z}^{d}\right|_{\mathbb{S}^{1}}$ and $\left.\mathcal{N}_{d}\right|_{\mathbb{S}^{1}}$ fixing 1 . We define

$$
\mathcal{G}_{\Gamma}:= \begin{cases}\mathcal{N}_{\Gamma} & \text { on } \overline{\mathcal{D}} \backslash \bigcup_{F} \Omega_{F}, \\ (\phi \circ \psi) \circ m_{-d} \circ(\phi \circ \psi)^{-1} & \text { on } \Omega_{F},\end{cases}
$$

where $m_{-d}(z)=\bar{z}^{d}$.

The map $\bar{z}^{d}$ has $d+1$ invariant rays in $\mathbb{D}$ connecting 0 with $e^{2 \pi i \cdot \frac{j}{d+1}}$ by radial line segments. We shall refer to these rays as internal rays. For each $\Omega_{F}$, we let $\mathscr{T}_{F}$ be the image of the union of these $d+1$ internal rays (under $\phi \circ \psi$ ), and we call the image of 0 the centre of $\Omega_{F}$. This graph $\mathscr{T}_{F}$ is a deformation retract of $\Pi_{F}$ fixing the ideal points. Thus, each ray of $\Omega_{F}$ lands exactly at a cusp where two circles of the circle packing touch. We define

$$
\mathscr{T}\left(\mathcal{G}_{\Gamma}\right):=\bigcup_{F} \overline{\mathscr{T}_{F}}
$$

and endow it with a simplicial structure such that the centres of the faces are vertices. Then $\mathscr{T}\left(\mathcal{G}_{\Gamma}\right)$ is the planar dual to the plane graph $\Gamma$. We will now see that $\mathcal{G}_{\Gamma}$ acts as a 'reflection' on each face of $\mathscr{T}\left(\mathcal{G}_{\Gamma}\right)$.

Lemma 3.22. Let $P$ be an open face of $\mathscr{T}\left(\mathcal{G}_{\Gamma}\right)$; then $\mathcal{G}_{\Gamma}$ is a homeomorphism sending $P$ to the interior of its complement.

Proof. Since $\Gamma$ is 2-connected, the complement of $\bar{P}$ is connected, so $P$ is a Jordan domain. Since $P$ contains no critical point and $\mathcal{G}_{\Gamma}(\partial P)=\partial P$, it follows that $\mathcal{G}_{\Gamma}$ sends $P$ homeomorphically to the interior of its complement. 
We shall see in the next section that this branched covering $\mathcal{G}_{\Gamma}$ is topologically conjugate to a critically fixed anti-rational map $R$ and this dual graph $\mathscr{T}\left(\mathcal{G}_{\Gamma}\right)$ is the Tischler graph associated with $R$.

\section{Critically Fixed Anti-rational Maps}

In this section, we shall show how critically fixed anti-rational maps are related to circle packings and kissing reflection groups. As a corollary of our study, we obtain a classification of critically fixed antirational maps in terms of the combinatorics of planar duals of Tischler graphs. A classification of these maps in terms of combinatorial properties of Tischler graphs was given independently by Geyer in a recent work [9]. For the purpose of establishing a dynamical correspondence between critically fixed anti-rational maps and kissing reflection groups, planar duals of Tischler graphs turn out to be a more natural combinatorial invariant.

The arguments employed in Subsection 4.1, where we investigate the structure (of planar duals) of Tischler graphs, parallel those used in the proof of [9, Theorem 4.3]. On the other hand, [9, §6] uses purely topological means to construct Thurston maps for the realisation part of the classification theorem; while we use the branched cover $\mathcal{G}_{\Gamma}$ of Subsection 3.5, which is cooked up from Nielsen maps of kissing reflection groups (see Subsection 4.2).

We define an anti-polynomial $P$ of degree $d$ as

$$
P(z)=a_{d} \bar{z}^{d}+a_{d-1} \bar{z}^{d-1}+\ldots+a_{0}
$$

where $a_{i} \in \mathbb{C}$ and $a_{d} \neq 0$. An anti-rational map $R$ of degree $d$ is the ratio of two anti-polynomials

$$
R(z)=\frac{P(z)}{Q(z)}
$$

where $P$ and $Q$ have no common zeroes and the maximum degree of $P$ and $Q$ is $d$. An anti-rational map of degree $d$ is an orientation-reversing branched covering of $\widehat{\mathbb{C}}$. It is said to be critically fixed if all of its critical points are fixed. The Julia set and Fatou set of anti-rational maps can be defined as in the rational setting.

\subsection{Tischler graph of critically fixed anti-rational maps}

Let $R$ be a critically fixed anti-rational map of degree $d$. Let $c_{1}, \cdots, c_{k}$ be the distinct critical points of $R$ and the local degree of $R$ at $c_{i}$ be $m_{i}(i=1, \cdots, k)$. Since $R$ has $(2 d-2)$ critical points counting multiplicity, we have that

$$
\sum_{i=1}^{k}\left(m_{i}-1\right)=2 d-2 \Longrightarrow \sum_{i=1}^{k} m_{i}=2 d+k-2 .
$$

Suppose that $U_{i}$ is the invariant Fatou component containing $c_{i}(i=1, \cdots, k)$. Then $U_{i}$ is a simply connected domain such that $\left.R\right|_{U_{i}}$ is conformally conjugate to $\left.\bar{z}^{m_{i}}\right|_{\mathbb{D}}$ [31, Theorem 9.3]. This defines internal rays in $U_{i}$ and $R$ maps the internal ray at angle $\theta \in \mathbb{R} / \mathbb{Z}$ to the one at angle $-m_{i} \theta \in \mathbb{R} / \mathbb{Z}$. It follows that there are $\left(m_{i}+1\right)$ fixed internal rays in $U_{i}$. A straightforward adaptation of [31, Theorem 18.10] now implies that all of these fixed internal rays land at repelling fixed points on $\partial U_{i}$.

We define the Tischler graph $\mathscr{T}$ of $R$ as the union of the closures of the fixed internal rays of $R$.

Lemma 4.1. $R$ has exactly $(d+2 k-1)$ distinct fixed points in $\widehat{\mathbb{C}}$ of which $(d+k-1)$ lie on the Julia set of $R$.

Proof. Note that $R$ has no neutral fixed point and exactly $k$ attracting fixed points. The count of the total number of fixed points of $R$ now follows from the Lefschetz fixed point theorem (see [22, Lemma 6.1]). 
Lemma 4.2. The fixed internal rays of $R$ land pairwise.

Proof. As $R$ is a local orientation-reversing diffeomorphism in a neighbourhood of the landing point of each internal ray, it follows that at most two distinct fixed internal rays may land at a common point.

Note that the total number of fixed internal rays of $R$ is

$$
\sum_{i=1}^{k}\left(m_{i}+1\right)=2(d+k-1),
$$

while there are only $(d+k-1)$ landing points available for these rays by Lemma 4.1. Since no more than two fixed internal rays can land at a common fixed point, the result follows.

In our setting, it is more natural to put a simplicial structure on $\mathscr{T}$ so that the vertices correspond to the critical points of $R$. We will refer to the repelling fixed points on $\mathscr{T}$ as the midpoints of the edges. To distinguish an edge of $\mathscr{T}$ from an arc connecting a vertex and a midpoint, we will call the latter an internal ray of $\mathscr{T}$.

Corollary 4.3. The valence of the critical point $c_{i}$ (as a vertex of $\left.\mathscr{T}\right)$ is $\left(m_{i}+1\right)(i=1, \cdots, k)$. The repelling fixed points of $R$ are in bijective correspondence with the edges of $\mathscr{T}$.

The proof of Lemma 4.2 implies the following (see [11, Corollary 6] for the same statement in the holomorphic setting).

Corollary 4.4. Each fixed point of $R$ lies on (the closure of) a fixed internal ray.

We are now ready to establish the key properties of the Tischler graph of a critically fixed anti-rational map that will be used in the combinatorial classification of such maps.

Lemma 4.5. The faces of $\mathscr{T}$ are Jordan domains.

Proof. Let $F$ be a face of the Tischler graph $\mathscr{T}$. Then a component of the ideal boundary $I(F)$ consists of a sequence of edges $e_{1}, \ldots, e_{m}$ of $\mathscr{T}$, oriented counterclockwise viewed from $F$. Note that a priori, $e_{i}$ may be equal to $e_{j}$ in $\mathscr{T}$ for different $i$ and $j$.

Consider the graph $T$ whose vertices are components of $\widehat{\mathbb{C}} \backslash \bar{F}$ and two vertices $U, V$ are connected by an edge if there is a path in $\widehat{\mathbb{C}} \backslash F$ connecting $U, V$ and not passing through other components. Then $T$ is a finite union of trees. Since each vertex (of $\mathscr{T}$ ) has valence at least 3 , we have that $e_{i} \neq e_{i+1}$. This also implies that two adjacent components $U, V$ either share a common boundary vertex or there exists an edge of $\mathscr{T}$ connecting them. Let $U$ be a component of $\widehat{\mathbb{C}} \backslash \bar{F}$. If $e_{i}, e_{j}$ are a pair of adjacent edges of $\partial U$ but $e_{i}, e_{j}$ are not adjacent on the ideal boundary $I(F)$, then there exists a component $V$ of $\widehat{\mathbb{C}} \backslash \bar{F}$ 'attached' to $U$ through the vertex $v=e_{i} \cap e_{j}$ (see Figure 4.1). Therefore, the number of pairs of adjacent edges of $\partial U$ that are not adjacent on the ideal boundary $I(F)$ equals to the valence of $U$ in $T$. Choose $U$ to be an end point of $T$ and let $S=\partial U$.

Since $R$ is orientation-reversing and each edge of $\mathscr{T}$ is invariant under the map, $R$ reflects the two sides near each open edge of $S$. Thus, there is a connected component $V$ of $R^{-1}(U)$ with $\partial V \cap \partial U \neq \emptyset$ and $V \cap F \neq \emptyset$. It is easy to see from the local dynamics of $\bar{z}^{m_{i}}$ at the origin that near a critical point, $R$ sends the region bounded by two adjacent edges of $\mathscr{T}$ to its complement. Therefore, by our choice of $U$, we have $\partial U \subseteq \partial V$. Since $\partial F$ is $R$-invariant, $V \cap \partial F=\emptyset$. Thus, $V \subseteq F$ and $V$ contains no critical points of $R$. As $U$ is a disk, the Riemann-Hurwitz formula now implies that $V$ is a disk and $R$ is a homeomorphism from $\partial V$ to $\partial U$. Therefore, $\partial U=\partial V$ and $V=F$; so $F$ is a Jordan domain.

In particular, we have the following.

Corollary 4.6. The Tischler graph $\mathscr{T}$ is connected.

In fact, the Tischler graph gives a topological model for the map $R$. 


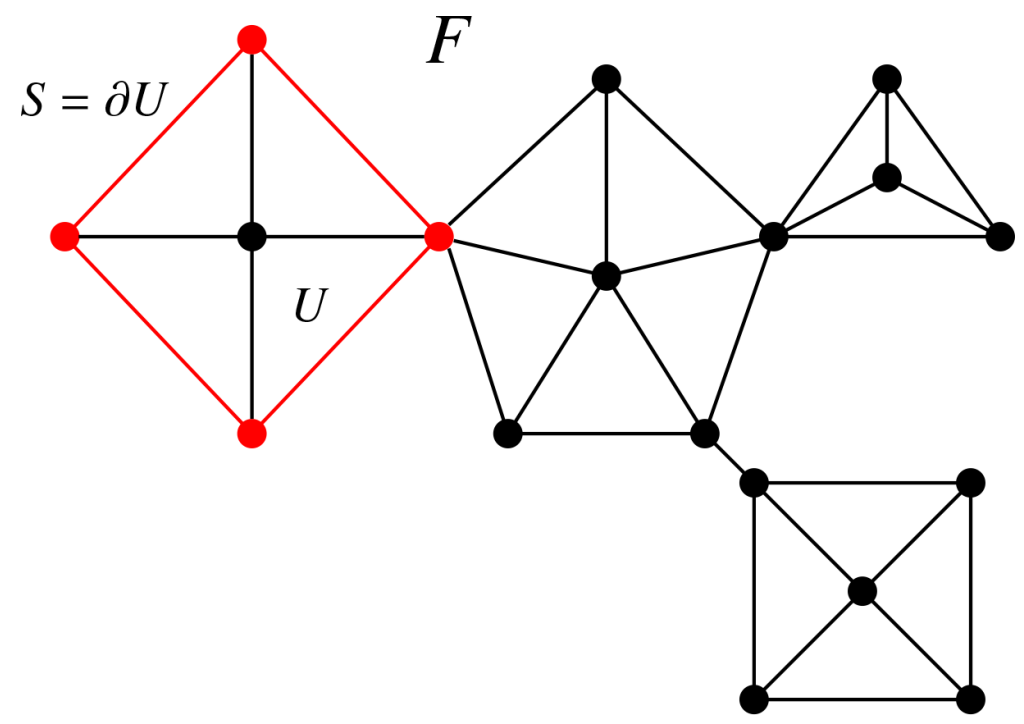

Figure 4.1. An a priori possible schematic of a component of the boundary of $F$.

Corollary 4.7. Let $F$ be a face of $\mathscr{T}$ and $F^{c}=\overline{\widehat{\mathbb{C}} \backslash F}$ be the closure of its complement. Then $R: \bar{F} \longrightarrow F^{c}$ is an orientation-reversing homeomorphism.

Lemma 4.8. Let $F_{1}, F_{2}$ be two faces of $\mathscr{T}$; then the boundaries share at most one edge.

Proof. Suppose that $F_{1}$ and $F_{2}$ share two or more edges. For $i=1,2$, let $\gamma_{i}$ be the hyperbolic geodesic arc in $\stackrel{\circ}{F}_{i}$ connecting the two midpoints of the edges and let $\gamma=\gamma_{1} \cup \gamma_{2}$. Since each vertex has valence at least 3, we see that $\gamma$ is essential in $\widehat{\mathbb{C}} \backslash V(\mathscr{T})=\widehat{\mathbb{C}} \backslash P(R)$ (that is, $\gamma$ is not homotopic to a point or a puncture of the surface $\widehat{\mathbb{C}} \backslash P(R)$ ), where $P(R)$ stands for the postcritical set of $R$ and $V(\mathscr{T})$ denotes the vertex set of the graph $\mathscr{T}$. By Corollary 4.7, there exists $\gamma^{\prime}$ homotopic to $\gamma$ in $\widehat{\mathbb{C}} \backslash P(R)$ such that $R: \gamma^{\prime} \longrightarrow \gamma$ is a homeomorphism. This gives a Thurston obstruction (more precisely, a Levy cycle) for the anti-rational map $R$ (see the discussion in Subsection 4.2 on Thurston's theory for rational maps), which is a contradiction. Hence, the supposition that $F_{1}$ and $F_{2}$ share two or more edges is false.

The following result, where we translate the above properties of the Tischler graph to a simple graphtheoretic property of its planar dual, plays a crucial role in the combinatorial classification of critically fixed anti-rational maps.

Lemma 4.9. Let $\Gamma$ be the planar dual of the Tischler graph $\mathscr{T}$ of a critically fixed anti-rational map $R$. Then $\Gamma$ is simple and 2-connected.

Proof. By Lemma 4.8, no two faces of $\mathscr{T}$ share two edges on their boundary. Hence, the dual graph contains no multi-edge. Again, as each face of $\mathscr{T}$ is a Jordan domain by Lemma 4.5, the dual graph contains no self-loop. Therefore, $\Gamma$ is simple.

The fact that each face of $\mathscr{T}$ is a Jordan domain also implies that the complement of the closure of each face is connected. So the dual graph $\Gamma$ remains connected upon deletion of any vertex. In other words, $\Gamma$ is 2 -connected.

\subsection{Constructing critically fixed anti-rational maps from graphs}

Let $\Gamma$ be a 2-connected simple plane graph and $G_{\Gamma}$ be an associated kissing reflection group. In Subsection 3.5, we constructed a topological branched covering $\mathcal{G}_{\Gamma}$ from the Nielsen map of $G_{\Gamma}$. In 
the remainder of this section, we will use this branched covering $\mathcal{G}_{\Gamma}$ to promote Lemma 4.9 to a characterisation of Tischler graphs of critically fixed anti-rational maps.

Proposition 4.10. A plane graph $\mathcal{T}$ is the Tischler graph of a critically fixed anti-rational map $R$ if and only if the dual (plane) graph $\Gamma$ is simple and 2-connected. Moreover, $R$ is topologically conjugate to $\mathcal{G}_{\Gamma}$.

We will first introduce some terminology. A postcritically finite branched covering (possibly orientation-reversing) of a topological 2 -sphere $\mathbb{S}^{2}$ is called a Thurston map. We denote the postcritical set of a Thurston map $f$ by $P(f)$. Two Thurston maps $f$ and $g$ are equivalent if there exist two orientationpreserving homeomorphisms $h_{0}, h_{1}:\left(\mathbb{S}^{2}, P(f)\right) \rightarrow\left(\mathbb{S}^{2}, P(g)\right)$ so that $h_{0} \circ f=g \circ h_{1}$, where $h_{0}$ and $h_{1}$ are isotopic relative to $P(f)$.

A set of pairwise disjoint, nonisotopic, essential, simple closed curves $\Sigma$ on $\mathbb{S}^{2} \backslash P(f)$ is called a curve system. A curve system $\Sigma$ is called $f$-stable if for every curve $\sigma \in \Sigma$, all of the essential components of $f^{-1}(\sigma)$ are homotopic rel $P(f)$ to curves in $\Sigma$. We associate to an $f$-stable curve system $\Sigma$ the Thurston linear transformation

$$
f_{\Sigma}: \mathbb{R}^{\Sigma} \longrightarrow \mathbb{R}^{\Sigma}
$$

defined as

$$
f_{\Sigma}(\sigma)=\sum_{\sigma^{\prime} \subseteq f^{-1}(\sigma)} \frac{1}{\operatorname{deg}\left(f: \sigma^{\prime} \rightarrow \sigma\right)}\left[\sigma^{\prime}\right]_{\Sigma},
$$

where $\sigma \in \Sigma$ and $\left[\sigma^{\prime}\right]_{\Sigma}$ denotes the element of $\Sigma$ isotopic to $\sigma^{\prime}$, if it exists. The curve system is called irrreducible if $f_{\Sigma}$ is irreducible as a linear transformation. It is said to be a Thurston obstruction if the spectral radius $\lambda\left(f_{\Sigma}\right) \geq 1$.

Similarly, an arc $\lambda$ in $\mathbb{S}^{2} \backslash P(f)$ is an embedding of $(0,1)$ in $\mathbb{S}^{2} \backslash P(f)$ with endpoints in $P(f)$. It is said to be essential if it is not contractible in $\mathbb{S}^{2}$ fixing the two endpoints. A set of pairwise nonisotopic essential arcs $\Lambda$ is called an arc system. The Thurston linear transformation $f_{\Lambda}$ is defined in a similar way, and we say that it is irreducible if $f_{\Lambda}$ is irreducible as a linear transformation.

The next proposition allows us to directly apply Thurston's topological characterisation theorem for rational maps (see [6, Theorem 1]) to the study of orientation-reversing Thurston maps (see [9, Theorem 3.9] for an intrinsic topological characterisation theorem for anti-rational maps). It is proved by considering the second iterate of Thurston's pullback map on the Teichmüller space of $\mathbb{S}^{2} \backslash P(f)$. The reader is referred to [6] for the definition of hyperbolic orbifold, but this is the typical case as any map with more than four postcritical points has hyperbolic orbifold.

Proposition 4.11. [24, Proposition 6.1] Let $f$ be an orientation-reversing Thurston map so that $f \circ f$ has hyperbolic orbifold. Then $f$ is equivalent to an anti-rational map if and only if $f \circ f$ is equivalent to a rational map if and only if $f \circ f$ has no Thurston obstruction. Moreover, if $f$ is equivalent to an anti-rational map, the map is unique up to Möbius conjugacy.

For a curve system $\Sigma$ (respectively an arc system $\Lambda$ ), we set $\widetilde{\Sigma}$ (respectively $\widetilde{\Lambda}$ ) as the union of those components of $f^{-1}(\Sigma)$ (respectively $f^{-1}(\Lambda)$ ) which are isotopic relative to $P(f)$ to elements of $\Sigma$ (respectively $\Lambda$ ). We will use $\Sigma \cdot \Lambda$ to denote the minimal intersection number between them. We will be using the following theorem excerpted and paraphrased from [38, Theorem 3.2].

Theorem 4.12. [38, Theorem 3.2] Let $f$ be an orientation-preserving Thurston map, $\Sigma$ an irreducible Thurston obstruction in $\left(\mathbb{S}^{2}, P(f)\right)$ and $\Lambda$ an irreducible arc system in $\left(\mathbb{S}^{2}, P(f)\right)$. Assume that $\Sigma$ intersect $\Lambda$ minimally; then either

$\circ \Sigma \cdot \Lambda=0 ;$ or

$\circ \Sigma \cdot \Lambda \neq 0$ and for each $\lambda \in \Lambda$, there is exactly one connected component $\lambda^{\prime}$ of $f^{-1}(\lambda)$ such that $\lambda^{\prime} \cap \widetilde{\Sigma} \neq \emptyset$. Moreover, the arc $\lambda^{\prime}$ is the unique component of $f^{-1}(\lambda)$ which is isotopic to an element of $\Lambda$. 
With these preparations, we are now ready to show that $\mathcal{G}_{\Gamma}$ is equivalent to an anti-rational map. The proof is similar to [24, Proposition 6.2].

Lemma 4.13. Let $\Gamma$ be a 2-connected simple plane graph. Then $\mathcal{G}_{\Gamma}$ is equivalent to a critically fixed anti-rational map $\mathcal{R}_{\Gamma}$.

Proof. It is easy to verify that $\mathcal{G}_{\Gamma} \circ \mathcal{G}_{\Gamma}$ has hyperbolic orbifold, except when $\Gamma=\Gamma_{d}$, in which case $\mathcal{G}_{\Gamma}$ has only two fixed critical points, each of which is fully branched and $\mathcal{G}_{\Gamma}$ is equivalent to $\bar{z}^{d}$. Thus, by Proposition 4.11, we will prove the lemma by showing that there is no Thurston obstruction for $\mathcal{G}_{\Gamma} \circ \mathcal{G}_{\Gamma}$.

We will assume that there is a Thurston obstruction $\Sigma$ and arrive at a contradiction. After passing to a subset, we may assume that $\Sigma$ is irreducible. Isotoping the curve system $\Sigma$, we may assume that $\Sigma$ intersects the graph $\mathscr{T}\left(\mathcal{G}_{\Gamma}\right)$ minimally (see Subsection 3.5 for the definition of $\mathscr{T}\left(\mathcal{G}_{\Gamma}\right)$ ). Let $\lambda$ be an edge in $\mathscr{T}\left(\mathcal{G}_{\Gamma}\right)$; then $\Lambda=\{\lambda\}$ is an irreducible arc system. Let $\widetilde{\Sigma}$ be the union of those components of $\mathcal{G}_{\Gamma}^{-2}(\Sigma)$ which are isotopic to elements of $\Sigma$.

We claim that $\widetilde{\Sigma}$ does not intersect $\mathcal{G}_{\Gamma}^{-2}(\lambda) \backslash \lambda$. Indeed, applying Theorem 4.12 , we are led to the following two cases. In the first case, we have $\Sigma \cdot \lambda=0$; hence, $\mathcal{G}_{\Gamma}^{-2}(\Sigma)$ cannot intersect $\mathcal{G}_{\Gamma}^{-2}(\lambda)$ and the claim follows. In the second case, since $\mathcal{G}_{\Gamma}(\lambda)=\lambda$, we conclude that $\lambda$ is the unique component of $\mathcal{G}_{\Gamma}^{-2}(\lambda)$ isotopic to $\lambda$. Thus, the only component of $\mathcal{G}_{\Gamma}^{-2}(\lambda)$ intersecting $\widetilde{\Sigma}$ is $\lambda$, so the claim follows.

Applying this argument on all the edges of $\mathscr{T}\left(\mathcal{G}_{\Gamma}\right)$, we conclude that $\widetilde{\Sigma}$ does not intersect $\mathcal{G}_{\Gamma}^{-2}\left(\mathscr{T}\left(\mathcal{G}_{\Gamma}\right)\right) \backslash \mathscr{T}\left(\mathcal{G}_{\Gamma}\right)$.

Let $F$ be a face of $\mathscr{T}\left(\mathcal{G}_{\Gamma}\right)$ with vertices of $\partial F$ denoted by $v_{1}, \ldots, v_{k}$ counterclockwise. Denote $e_{i} \subseteq \partial F$ be the open edge connecting $v_{i}, v_{i+1}$. We claim that the graph obtained by removing the boundary edges of $F$

$$
\mathscr{T}_{F}:=\mathscr{T}\left(\mathcal{G}_{\Gamma}\right) \backslash \bigcup_{i=1}^{k} e_{i}
$$

is still connected. Since $\mathscr{T}\left(\mathcal{G}_{\Gamma}\right)$ is connected, any vertex $v$ is connected to the set $\left\{v_{1}, \ldots, v_{k}\right\}$ in $\mathscr{T}_{F}$. Thus, it suffices to show that $v_{i}$ and $v_{i+1}$ are connected in $\mathscr{T}_{F}$ for all $i$. As the planar dual $\Gamma$ of $\mathscr{T}\left(\mathcal{G}_{\Gamma}\right)$ is 2-connected and has no self-loop, each face of $\mathscr{T}\left(\mathcal{G}_{\Gamma}\right)$ is a Jordan domain. Hence, there exists a face $F_{i} \neq F$ of $\mathscr{T}\left(\mathcal{G}_{\Gamma}\right)$ sharing $e_{i}$ on the boundary with $F$. Moreover, since $\Gamma$ has no multi-edge, the boundaries of any two faces of $\mathscr{T}\left(\mathcal{G}_{\Gamma}\right)$ intersect at most at one edge. Thus, $\partial F_{i} \cap \partial F=e_{i} \cup\left\{v_{i}, v_{i+1}\right\}$. Hence, $v_{i}$ and $v_{i+1}$ are connected by a path in $\partial F_{i} \backslash e_{i} \subseteq \mathscr{T}_{F}$, and this proves the claim.

Therefore, by Lemma 3.22, we deduce that

$$
\left(\mathcal{G}_{\Gamma}^{-1}\left(\mathscr{T}\left(\mathcal{G}_{\Gamma}\right)\right) \backslash \mathscr{T}\left(\mathcal{G}_{\Gamma}\right)\right) \cup V\left(\mathscr{T}\left(\mathcal{G}_{\Gamma}\right)\right)
$$

is connected. Thus, $\left(\mathcal{G}_{\Gamma}^{-2}\left(\mathscr{T}\left(\mathcal{G}_{\Gamma}\right)\right) \backslash \mathscr{T}\left(\mathcal{G}_{\Gamma}\right)\right) \cup V\left(\mathscr{T}\left(\mathcal{G}_{\Gamma}\right)\right)$ is a connected graph containing the postcritical set $P\left(\mathcal{G}_{\Gamma} \circ \mathcal{G}_{\Gamma}\right)=V\left(\mathscr{T}\left(\mathcal{G}_{\Gamma}\right)\right)$. This forces $\Sigma$ to be empty, which is a contradiction.

Therefore, $\mathcal{G}_{\Gamma}$ is equivalent to an anti-rational map $\mathcal{R}_{\Gamma}$. Since $\mathcal{G}_{\Gamma}$ is critically fixed, so is $\mathcal{R}_{\Gamma}$.

Let $h_{0}, h_{1}:\left(\mathbb{S}^{2}, P\left(\mathcal{G}_{\Gamma}\right)\right) \rightarrow\left(\mathbb{S}^{2}, P\left(\mathcal{R}_{\Gamma}\right)\right)$ be two orientation-preserving homeomorphisms so that $h_{0} \circ \mathcal{G}_{\Gamma}=\mathcal{R}_{\Gamma} \circ h_{1}$ where $h_{0}$ and $h_{1}$ are isotopic relative to $P\left(\mathcal{G}_{\Gamma}\right)=V\left(\mathscr{T}\left(\mathcal{G}_{\Gamma}\right)\right)$.

We now use a standard pullback argument to prove the following.

Lemma 4.14. $h_{0}\left(\mathscr{T}\left(\mathcal{G}_{\Gamma}\right)\right)$ is isotopic to the Tischler graph $\mathscr{T}\left(\mathcal{R}_{\Gamma}\right)$.

Proof. Let $\alpha$ be an edge of $\mathscr{T}\left(\mathcal{G}_{\Gamma}\right)$; then $\mathcal{G}_{\Gamma}(\alpha)=\alpha$. Consider the sequence of homeomorphisms $h_{i}$ with

$$
h_{i-1} \circ \mathcal{G}_{\Gamma}=\mathcal{R}_{\Gamma} \circ h_{i}
$$

Each $h_{i}$ is normalised so that it carries $P\left(\mathcal{G}_{\Gamma}\right)$ to $P\left(\mathcal{R}_{\Gamma}\right)$. Note by induction, $\beta_{i}=h_{i}(\alpha)$ is isotopic to $\beta_{0}$ relative to the endpoints. Applying isotopy, we may assume that there is a decomposition $\beta_{0}=\beta_{0}^{-}+\gamma_{0}+\beta_{0}^{+}$, 
where $\beta_{0}^{ \pm}$are two internal rays and $\gamma_{0}$ does not intersect any invariant Fatou component. Then every $\beta_{i}=\beta_{i}^{-}+\gamma_{i}+\beta_{i}^{+}$has such a decomposition, too.

Note that $\mathcal{R}_{\Gamma}\left(\gamma_{i+1}\right)=\gamma_{i}$ and $\mathcal{R}_{\Gamma}: \gamma_{i+1} \rightarrow \gamma_{i}$ is injective for each $i$. Since $\mathcal{R}_{\Gamma}$ is hyperbolic and the curves $\gamma_{i}$ are uniformly bounded away from the postcritical point set of $\mathcal{R}_{\Gamma}$, we conclude that the sequence of curves $\left\{\gamma_{i}\right\}$ shrinks to a point. Thus, if $\beta$ is a limit of $\beta_{i}$ in the Hausdorff topology, then $\beta$ is a union of two internal rays and it is isotopic to $\beta_{0}$. Therefore, $h_{0}(\alpha)$ is isotopic to an edge of $\mathscr{T}\left(\mathcal{R}_{\Gamma}\right)$.

Since this is true for every edge of $\mathscr{T}\left(\mathcal{G}_{\Gamma}\right)$ and $h_{0}$ is an orientation-preserving homeomorphism, by counting the valence at every critical point, we conclude that $h_{0}\left(\mathscr{T}\left(\mathcal{G}_{\Gamma}\right)\right)$ is isotopic to $\mathscr{T}\left(\mathcal{R}_{\Gamma}\right)$.

Therefore, after performing isotopy, we may assume that $h_{0}$ restricts to a homeomorphism between the graphs $h_{0}: \mathscr{T}\left(\mathcal{G}_{\Gamma}\right) \longrightarrow \mathscr{T}\left(\mathcal{R}_{\Gamma}\right)$. By lifting, $h_{1}: \mathscr{T}\left(\mathcal{G}_{\Gamma}\right) \longrightarrow \mathscr{T}\left(\mathcal{R}_{\Gamma}\right)$ is also a homeomorphism. Let $F$ be a face of $\Gamma$. By our construction of $\mathcal{G}_{\Gamma}$ (see Equation 3.2), $\mathcal{G}_{\Gamma}$ is conjugate to $\bar{z}^{d_{F}}$ on the corresponding component $\Omega_{F}$, where $d_{F}+1$ is the number of edges in the ideal boundary of the face $F$. Since $\mathscr{T}\left(\mathcal{G}_{\Gamma}\right)$ is constructed by taking the union over all faces of the fixed internal rays under such conjugacies (see Equation 3.3), the dynamics of $\mathcal{G}_{\Gamma}$ and $\mathcal{R}_{\Gamma}$ on each edge of $\mathscr{T}\left(\mathcal{G}_{\Gamma}\right)$ and $\mathscr{T}\left(\mathcal{R}_{\Gamma}\right)$ are conjugate. Therefore, after further isotoping $h_{0}$, we may assume that

$$
h_{0}=h_{1}: \mathscr{T}\left(\mathcal{G}_{\Gamma}\right) \longrightarrow \mathscr{T}\left(\mathcal{R}_{\Gamma}\right)
$$

gives a conjugacy between $\mathcal{G}_{\Gamma}$ and $\mathcal{R}_{\Gamma}$ from $\mathscr{T}\left(\mathcal{G}_{\Gamma}\right)$ to $\mathscr{T}\left(\mathcal{R}_{\Gamma}\right)$. Hence, both $h_{0}$ and $h_{1}$ send a face of $\mathscr{T}\left(\mathcal{G}_{\Gamma}\right)$ homomorphically to the corresponding face of $\mathscr{T}\left(\mathcal{R}_{\Gamma}\right)$.

As an application of the above, we will show that the dynamics on the limit set and Julia set are topologically conjugate (cf. [24, Theorem 6.11]).

Lemma 4.15. There is a homeomorphism $h: \Lambda\left(G_{\Gamma}\right) \longrightarrow \mathcal{J}\left(\mathcal{R}_{\Gamma}\right)$ satisfying $h \circ \mathcal{G}_{\Gamma}=\mathcal{R}_{\Gamma} \circ h$.

Proof. Let $P^{0}$ consist of the points of tangency of the circle packing, $P^{i+1}:=\mathcal{G}_{\Gamma}^{-1}\left(P^{i}\right)$ and $P^{\infty}:=\bigcup_{i=0}^{\infty} P^{i}$. Then $P^{\infty}$ corresponds to the $G_{\Gamma}$ orbit of the cusps and hence is dense in $\Lambda\left(G_{\Gamma}\right)$. Let $Q^{0}:=h_{0}\left(P^{0}\right)$, which is also the set of repelling fixed points of $\mathcal{R}_{\Gamma}$ and $Q^{i+1}:=\mathcal{R}_{\Gamma}^{-1}\left(Q^{i}\right)$. Then $Q^{\infty}:=\bigcup_{i=0}^{\infty} Q^{i}$ is dense in $\mathcal{J}\left(\mathcal{R}_{\Gamma}\right)$.

Recall that for each face $F$ of $\Gamma$, the dynamics of $\mathcal{G}_{\Gamma}$ (respectively of $\mathcal{R}_{\Gamma}$ ) on the corresponding component of $\Omega\left(G_{\Gamma}\right)$ (respectively on the corresponding critically fixed Fatou component of $\mathcal{R}_{\Gamma}$ ) can be uniformised to $\bar{z}^{d}$ on $\mathbb{D}$. Let $\Pi$ be the closed ideal $d+1$-gon in $\mathbb{D}$ with ideal vertices at the fixed points of $\bar{z}^{d}$ on $\mathbb{S}^{1}$. Let $\mathcal{L}_{\mathcal{G}, F}$ and $\mathcal{L}_{\mathcal{R}, F}$ be the image of $\Pi$ under the uniformising map in the dynamical plane of $\mathcal{G}_{\Gamma}$ and $\mathcal{R}_{\Gamma}$ respectively and $\mathcal{L}_{\mathcal{G}}$ and $\mathcal{L}_{\mathcal{R}}$ be the union over all faces.

Let $\mathcal{E}^{0}:=\widehat{\mathbb{C}} \backslash \overline{\mathcal{L}_{\mathcal{G}}}$ and $\mathcal{H}^{0}:=\widehat{\mathbb{C}} \backslash \overline{\mathcal{L}_{\mathcal{R}}}$. Inductively, let $\mathcal{E}^{i+1}:=\mathcal{G}_{\Gamma}^{-1}\left(\mathcal{E}^{i}\right)$ and $\mathcal{H}^{i+1}:=\mathcal{R}_{\Gamma}^{-1}\left(\mathcal{H}^{i}\right)$. Note that these sets are the analogues of $\mathcal{D}^{i}$ for kissing reflection groups. Since $\mathcal{G}_{\Gamma}$ (respectively $\mathcal{R}_{\Gamma}$ ) sends a face of $\mathscr{T}\left(\mathcal{G}_{\Gamma}\right)$ (respectively a face of $\mathscr{T}\left(\mathcal{R}_{\Gamma}\right)$ ) to its complement univalently, our construction guarantees that $\overline{\mathcal{E}^{1}} \subseteq \overline{\mathcal{E}^{0}}$ and $\overline{\mathcal{H}^{1}} \subseteq \overline{\mathcal{H}^{0}}$. Inductively, we see that $\left\{\overline{\mathcal{E}^{i}}\right\}$ and $\left\{\overline{\mathcal{H}^{i}}\right\}$ are nested sequences of closed sets. Thus, we have

$$
\Lambda\left(G_{\Gamma}\right)=\bigcap_{i=0}^{\infty} \overline{\mathcal{E}^{i}}
$$

and

$$
\mathcal{J}\left(\mathcal{R}_{\Gamma}\right)=\bigcap_{i=0}^{\infty} \overline{\mathcal{H}^{i}}
$$

Since $\mathcal{R}_{\Gamma}$ is hyperbolic and $\mathcal{H}^{i}$ contains no critical value, the diameters of the components of $\mathcal{H}^{i} \cap \mathcal{J}\left(\mathcal{R}_{\Gamma}\right)$ shrink to 0 uniformly. On the other hand, since $\mathcal{G}_{\Gamma}=\mathcal{N}_{\Gamma}$ on $\Lambda\left(G_{\Gamma}\right)$, the diameters of the components of $\mathcal{E}^{i} \cap \Lambda\left(G_{\Gamma}\right)$ shrink to 0 uniformly by Lemma 3.3. 
After isotoping $h_{0}$, we may assume that $h_{0}\left(\mathcal{E}^{0}\right)=\mathcal{H}^{0}$. We consider the pullback sequence $\left\{h_{i}\right\}$ with

$$
h_{i-1} \circ \mathcal{G}_{\Gamma}=\mathcal{R}_{\Gamma} \circ h_{i},
$$

where each $h_{i}$ carries $P\left(\mathcal{G}_{\Gamma}\right)$ to $P\left(\mathcal{R}_{\Gamma}\right)$. Since we have assumed $h_{0}=h_{1}$ on $P^{0}$, inductively, we have $h_{j}=h_{i}$ on $P_{i}$ and $h_{j}\left(P^{i}\right)=Q^{i}$ for all $j \geq i$. We define $h(x):=\lim h_{i}(x)$ for $x \in P^{\infty}$.

Then a similar argument as in the proof of Proposition 3.21 implies that $h$ is uniformly continuous on $P^{\infty}$. Indeed, given $\epsilon>0$, we can choose $N$ so that the diameter of any component of $\mathcal{H}^{N} \cap \mathcal{J}\left(\mathcal{R}_{\Gamma}\right)$ is less than $\epsilon$. We choose $\delta$ so that any two nonadjacent components of $\mathcal{E}^{N} \cap \Lambda\left(G_{\Gamma}\right)$ are at least $\delta$ distance away. If $x, y \in P^{\infty}$ are at most $\delta$ distance apart, they lie in two adjacent components of $\mathcal{E}^{N}$, so $h_{j}(x), h_{j}(y)$ lie in two adjacent components of $\mathcal{H}^{N}$ for all $j \geq N$. Hence, $d\left(h_{j}(x), h_{j}(y)\right)<2 \epsilon$.

Since $P^{\infty}$ and $Q^{\infty}$ are dense on $\Lambda\left(G_{\Gamma}\right)$ and $\mathcal{J}\left(\mathcal{R}_{\Gamma}\right)$ (respectively), we get a unique continuous extension

$$
h: \Lambda\left(G_{\Gamma}\right) \longrightarrow \mathcal{J}\left(\mathcal{R}_{\Gamma}\right) .
$$

Applying the same argument on the sequence $\left\{h_{j}^{-1}\right\}$, we get the continuous inverse of $h$. Thus, $h$ is a homeomorphism. Since $h$ conjugates $\mathcal{G}_{\Gamma}$ to $\mathcal{R}_{\Gamma}$ on $P^{\infty}$, it also conjugates $\mathcal{G}_{\Gamma}$ to $\mathcal{R}_{\Gamma}$ on $\Lambda\left(G_{\Gamma}\right)$.

We are now ready to prove Proposition 4.10 (cf. [24, Proposition 6.13]).

Proof of Proposition 4.10. By Lemma 4.9, the dual graph of a Tischler graph (of a critically fixed antirational map) is 2-connected simple and plane.

Conversely, given any 2-connected simple plane graph $\Gamma$, by Lemma 4.13 and Lemma 4.14 we can construct a critically fixed anti-rational map $\mathcal{R}_{\Gamma}$ whose Tischler graph is the planar dual of $\Gamma$.

It remains to show that $\mathcal{G}_{\Gamma}$ and $\mathcal{R}_{\Gamma}$ are topologically conjugate. Let $h$ be the topological conjugacy on $\Lambda\left(G_{\Gamma}\right)$ produced in Lemma 4.15. Let $U$ be a critically fixed component $\mathcal{G}_{\Gamma}$. Then by construction, there exists $\Phi: \mathbb{D} \longrightarrow U$ conjugating $m_{-d}(z)=\bar{z}^{d}$ to $\mathcal{G}_{\Gamma}$. The map extends to a semiconjugacy between $\mathbb{S}^{1}$ and $\partial U$.

Similarly, let $V$ be the corresponding critically fixed Fatou component of $\mathcal{R}_{\Gamma}$. Then we have $\Psi$ : $\mathbb{D} \longrightarrow V$ conjugating $m_{-d}(z)=\bar{z}^{d}$ to $\mathcal{R}_{\Gamma}$, which extends to a semiconjugacy. Note that there are $d+1$ different choices of such a conjugacy, and we may choose one so that $h \circ \Phi=\Psi$ on $\mathbb{S}^{1}$. Thus, we can extend the topological conjugacy $h$ to all of $U$ by setting $h:=\Psi \circ \Phi^{-1}$.

In this way, we obtain a homeomorphic extension of $h$ to all critically fixed components of $\mathcal{G}_{\Gamma}$. Lifting these extensions to all of the preimages of the critically fixed components of $\mathcal{G}_{\Gamma}$, we get a map defined on $\widehat{\mathbb{C}}$. Since the diameters of the preimages of the critically fixed components (of $\mathcal{G}_{\Gamma}$ ) as well as the corresponding Fatou components (of $\mathcal{R}_{\Gamma}$ ) go to 0 uniformly, we conclude that all of these extensions paste together to yield a global homeomorphism, which is our desired topological conjugacy.

In light of Proposition 4.10, we see that the association of the isomorphism class of a 2-connected simple plane graph to the Möbius conjugacy class of a critically fixed anti-rational map is well defined and surjective. To verify that this is indeed injective, we remark that if two (plane) graphs $\Gamma$ and $\Gamma^{\prime}$ are isomorphic as plane graphs, then the associated Tischler graphs are also isomorphic as plane graphs. This means that the corresponding pair of critically fixed anti-rational maps are Thurston equivalent. Thus, by Thurston's rigidity result, they are Möbius conjugate.

As in the kissing reflection group setting, we define a geometric mating of two (anti-)polynomials as follows.

Definition 4.16. We say that a rational map (or an anti-rational map) $R$ is a geometric mating of two polynomials (or two anti-polynomials) $P^{ \pm}$with connected Julia sets if we have

$\circ$ a decomposition of the Fatou set $\mathcal{F}(R)=\mathcal{F}^{+} \sqcup \mathcal{F}^{-}$with $\mathcal{J}(R)=\partial \mathcal{F}^{+}=\partial \mathcal{F}^{-}$; and

$\circ$ two continuous surjections from the filled Julia sets $\psi^{ \pm}: \mathcal{K}\left(P^{ \pm}\right) \rightarrow \overline{\mathcal{F}^{ \pm}}$that are conformal between Int $\mathcal{K}\left(P^{ \pm}\right)$and $\mathcal{F}^{ \pm}$ 
so that

$$
\psi^{ \pm} \circ P^{ \pm}=R \circ \psi^{ \pm}
$$

As a corollary, we have the following.

\section{Corollary 4.17.}

1. A critically fixed anti-rational map $R$ is an anti-polynomial if and only if the dual graph $\Gamma$ of $\mathscr{T}(R)$ is outerplanar.

2. A critically fixed anti-rational map $R$ is a geometric mating of two anti-polynomials if and only if the dual graph $\Gamma$ of $\mathscr{T}(R)$ is Hamiltonian.

3. A critically fixed anti-rational map $R$ has a gasket Julia set if and only if the dual graph $\Gamma$ of $\mathscr{T}(R)$ is 3-connected.

Proof. The first statement follows from Proposition 4.10 as the graph $\Gamma$ is outerplanar if and only if there is a vertex in the dual graph $\Gamma^{\vee}=\mathscr{T}(R)$ with maximal valence if and only if $R$ has a fully branched fixed critical point.

For the second statement, let $\Gamma$ be a 2-connected simple plane graph. Let $G_{\Gamma}$ be an associated kissing reflection group and $\mathcal{G}_{\Gamma}$ and $\mathcal{R}_{\Gamma}$ be the associated branched covering and anti-rational map. By Proposition $4.10, \mathcal{G}_{\Gamma}$ and $\mathcal{R}_{\Gamma}$ are topologically conjugated by $h$.

If $\mathcal{R}_{\Gamma}$ is a geometric mating of two anti-polynomials $P^{ \pm}$which are necessarily critically fixed, then the conjugacy gives a decomposition of $\Omega\left(G_{\Gamma}\right)=\Omega^{+} \sqcup \Omega^{-}$. It is not hard to check that the $\Gamma$-actions on $\overline{\Omega^{ \pm}}$are conjugate to the actions of the function kissing reflection groups $G^{ \pm}$on their filled limit sets, where $G^{ \pm}$correspond to $P^{ \pm}$. Thus, $G_{\Gamma}$ is a geometric mating of the two function kissing reflection groups $G^{ \pm}$, so $\Gamma$ is Hamiltonian by Proposition 3.21.

Conversely, if $\Gamma$ is Hamiltonian, then $G_{\Gamma}$ is a geometric mating of two function kissing reflection groups $G^{ \pm}$and we get a decomposition of $\Omega\left(G_{\Gamma}\right)$ by Proposition 3.21. The conjugacy $h$ transports this decomposition to the anti-rational map setting. One can now check directly that $\mathcal{R}_{\Gamma}$ is a geometric mating of the anti-polynomials $P^{ \pm}$that are associated to the function kissing reflection groups $G^{ \pm}$.

The third statement follows immediately from Propositions 4.10 and 3.10.

Remark 4.18. The Hubbard tree of a critically fixed anti-polynomial $P$ is a strict subset of $\mathscr{T}(P) \cap \mathcal{K}(P)$, where $\mathcal{K}(P)$ is the filled Julia set of $P$ (cf. [20, §5]).

Remark 4.19. It is not hard to see that the existence of a Hamiltonian cycle for $\mathscr{T}(R)^{\vee}$ is equivalent to the existence of an equator for $R$ in the sense of [30, Definition 4.1]. This gives an alternative proof of the second statement of Corollary 4.17 (cf. [30, Theorem 4.2]).

\subsection{Dynamical correspondence}

Let $G_{\Gamma}$ and $\mathcal{R}_{\Gamma}$ be a kissing reflection group and a critically fixed anti-rational map associated to a 2 -connected simple plane graph $\Gamma$. Here we summarise the correspondence between various dynamical objects associated with the group and the anti-rational map.

- Markov partitions for limit and Julia sets: The associated circle packing $\mathcal{P}$ gives a Markov partition for the group dynamics on the limit set (more precisely, for the action of the Nielsen map $\mathcal{N}_{\Gamma}$ on $\Lambda\left(G_{\Gamma}\right)$ ). On the other side, the faces of the Tischler graph determine a Markov partition for the action of $\mathcal{R}_{\Gamma}$ on the Julia set. The topological conjugacy respects this pair of Markov partitions and the itineraries of points with respect to the corresponding Markov partitions.

- Cusps and repelling fixed points: The orbits of parabolic fixed points of $G_{\Gamma}$ bijectively correspond to pairs of adjacent vertices or, equivalently, to the edges of $\Gamma$. On the other side, the repelling fixed points of $\mathcal{R}_{\Gamma}$ are in bijective correspondence with the edges of the Tischler graph $\mathscr{T}=\Gamma^{\vee}$ and thus also with the edges of $\Gamma$. They are naturally identified by the topological conjugacy. 
○ $\sigma$-Invariant curves and 2-cycles: Each $\sigma$-invariant simple closed curve on the conformal boundary $\partial \mathcal{M}\left(G_{\Gamma}\right)$ corresponds to a pair of nonadjacent vertices on a face of $\Gamma$ (see the discussion on pinching deformations after Proposition 3.17 for the definition of the involution $\sigma$ ). On the other side, the above Markov partition for $\left.\mathcal{R}_{\Gamma}\right|_{\mathcal{J}(\mathcal{R}(\Gamma))}$ shows that each 2-cycle on the ideal boundary of an invariant Fatou component corresponds to a pair of nonadjacent vertices on the associated face of $\Gamma$. These $\sigma$-invariant curves and 2-cycles are naturally identified by the topological conjugacy. We remark that it is possible that a $\sigma$-invariant curve yields an accidental parabolic element, in which case the corresponding 2-cycle on the ideal boundary of the invariant Fatou component coalesces and gives rise to a repelling fixed point of $\mathcal{R}_{\Gamma}$. This happens if and only if the two corresponding vertices are adjacent in $\Gamma$.

- Question mark conjugacy: If we choose our group $G_{\Gamma}$ so that each conformal boundary is the double of a suitable regular ideal polygon, then the restriction of the homeomorphism $h$ of Lemma 4.15 between the boundary of an invariant Fatou component and the boundary of the corresponding component of $\Omega\left(G_{\Gamma}\right)$ gives a homeomorphism between the ideal boundaries $\phi: \mathbb{S}^{1} \longrightarrow \mathbb{S}^{1}$ that conjugates $\bar{z}^{e}$ to the Nielsen map $\mathcal{N}_{e}$ of the regular ideal $(e+1)$-gon reflection group. This conjugacy $\phi$ is a generalisation of the Minkowski question mark function (see [21, §3.2, §5.4.2] [24, Remark 9.1]).

- Function groups and anti-polynomials: If $G_{\Gamma}$ is a function kissing reflection group (of rank $\left.d+1\right)$, then it can be constructed by pinching a $\sigma$-invariant multicurve $\alpha$ on one component of $\partial \mathcal{M}\left(\mathbf{G}_{d}\right)$ (recall that $\mathbf{G}_{d}$ is the regular ideal polygon group of rank $d+1$ ). Under the natural orientationreversing identification of the two components of $\partial \mathcal{M}\left(\mathbf{G}_{d}\right)$, the multicurve $\alpha$ gives a $\sigma$-invariant multicurve $\alpha^{\prime}$ on the component of $\partial \mathcal{M}\left(G_{\Gamma}\right)$ associated to the $G_{\Gamma}$-invariant domain $\Omega_{0} \subseteq \Omega\left(G_{\Gamma}\right)$. The multicurve $\alpha^{\prime}$ consists precisely of the simple closed curves corresponding to the accidental parabolics of $G_{\Gamma}$. On the other side, the corresponding 2-cycles on the ideal boundary of the unbounded Fatou component (that is, the basin of infinity) generate the lamination for the Julia set of the anti-polynomial $R_{\Gamma}$.

- Quasi-Fuchsian closure and mating: If $G_{\Gamma}$ lies in the closure of the quasi-Fuchsian deformation space of $\mathbf{G}_{d}$, then $G_{\Gamma}$ is obtained by pinching two nonparallel $\sigma$-invariant multicurves $\alpha^{+}$and $\alpha^{-}$ on the two components of $\partial \mathcal{M}\left(\mathbf{G}_{d}\right)$; equivalently, $G_{\Gamma}$ is a mating of two function kissing reflection groups $G^{+}$and $G^{-}$. On the other side, the critically fixed anti-rational map $\mathcal{R}_{\Gamma}$ is a mating of the critically fixed anti-polynomials $P^{+}$and $P^{-}$, which correspond to the groups $G^{+}$and $G^{-}$(respectively). The topological conjugacy between $\left.\mathcal{N}_{\Gamma}\right|_{\Lambda\left(G_{\Gamma}\right)}$ and $\left.\mathcal{R}_{\Gamma}\right|_{\mathcal{J}\left(\mathcal{R}_{\Gamma}\right)}$ is induced by the circle homeomorphism that conjugates $\mathcal{N}_{d}$ to $\bar{z}^{d}$.

\subsection{Mating of two anti-polynomials}

In this subsection, we shall discuss the converse question of mateablity in terms of laminations. Let

$$
P(z)=\bar{z}^{d}+a_{d-2} \bar{z}^{d-2}+a_{d-3} \bar{z}^{d-3}+\ldots+a_{0}
$$

be a monic centred anti-polynomial with connected Julia set. We denote the filled Julia set by $\mathcal{K}(P)$. There is a unique Böttcher coordinate

$$
\psi: \mathbb{C} \backslash \overline{\mathbb{D}} \longrightarrow \mathbb{C} \backslash \mathcal{K}(P)
$$

with derivative 1 at infinity that conjugates $\bar{z}^{d}$ to $P$. We shall call a monic centred anti-polynomial equipped with this preferred Böttcher coordinate a marked anti-polynomial. Note that when the Julia set is connected, this marking is equivalent to an affine conjugacy class of anti-polynomials together with a choice of Böttcher coordinate at infinity. If we further assume that the Julia set is locally connected, the map $\psi$ extends to a continuous semi-conjugacy $\psi: \mathbb{S}^{1} \longrightarrow \mathcal{J}(P)$ between $\bar{z}^{d}$ and $P$. This semiconjugacy gives rise to a $\bar{z}^{d}$-invariant lamination on the circle $\mathbb{S}^{1}$ for the marked anti-polynomial. The 
coordinate on $\mathbb{S}^{1} \cong \mathbb{R} / \mathbb{Z}$ will be called external angle. The image of $\left\{r e^{2 \pi i \theta} \in \mathbb{C}: r \geq 1\right\}$ under $\psi$ will be called an external ray at angle $\theta$ and will be denoted by $R(\theta)$. If $x \in \mathcal{J}(P)$ is the intersection of two external rays, then $x$ is a cut-point of $\mathcal{J}(P)$ (equivalently, a cut-point of $\mathcal{K}(P)$ ).

If we denote $\mathbb{C}=\mathbb{C} \cup\left\{(\infty, w): w \in \mathbb{S}^{1}\right\}$ as the complex plane together with the circle at infinity, then a marked anti-polynomial extends continuously to (C) by the formula $P(\infty, w)=\left(\infty, \bar{w}^{d}\right)$.

Let $P$ and $Q$ be two marked degree $d$ anti-polynomials. To distinguish the two domains (C), we denote them by $\mathbb{C}_{P}$ and $\mathbb{C}_{Q}$. The quotient

$$
\mathbb{S}_{P, Q}^{2}:=\left(\mathbb{C}_{P} \cup \mathbb{C}_{Q}\right) /\left\{\left(\infty_{P}, w\right) \sim\left(\infty_{Q}, \bar{w}\right)\right\}
$$

defines a topological sphere. Denote the equivalence class of $\left(\infty_{P}, w\right)$ by $(\infty, w)$ and define the equator of $\mathbb{S}_{P, Q}^{2}$ to be the set $\left\{(\infty, w): w \in \mathbb{S}^{1}\right\}$. The formal mating of $P$ and $Q$ is the degree $d$ branched cover $P \Perp Q: \mathbb{S}_{f, g}^{2} \rightarrow \mathbb{S}_{f, g}^{2}$ defined by the formula

$$
(P \Perp Q)(z)= \begin{cases}P(z) & z \in \mathbb{C}_{P}, \\ \left(\infty, \bar{z}^{d}\right) & \text { for }(\infty, z), \\ Q(z) & z \in \mathbb{C}_{Q} .\end{cases}
$$

Suppose that $P$ and $Q$ have connected and locally connected Julia sets. The closure in $\mathbb{S}_{P, Q}^{2}$ of the external ray at angle $\theta$ in $\mathbb{C}_{P}$ is denoted $R_{P}(\theta)$ and, likewise, the closure of the external ray at angle $\theta$ in $\mathbb{C}_{Q}$ is denoted $R_{Q}(\theta)$. Then $R_{P}(\theta)$ and $R_{Q}(-\theta)$ are rays in $\mathbb{S}_{P, Q}^{2}$ that share a common endpoint $\left(\infty, e^{2 \pi i \theta}\right)$. The extended external ray at angle $\theta$ in $\mathbb{S}_{P, Q}^{2}$ is defined as $R(\theta):=R_{P}(\theta) \cup R_{Q}(-\theta)$. Each extended external ray intersects each of $\mathcal{K}(P), \mathcal{K}(Q)$ and the equator at exactly one point.

We define the ray equivalence $\sim_{\text {ray }}$ as the smallest equivalence relation on $\mathbb{S}_{P, Q}^{2}$ so that two points $x, y \in \mathbb{S}_{P, Q}^{2}$ are equivalent if there exists $\theta$ so that $x, y \in R(\theta)$. A ray equivalence class $\gamma$ can be considered as an embedded graph: the vertices are the points in $\gamma \cap(\mathcal{K}(P) \cup \mathcal{K}(Q))$ and the edges are the external extended rays in $\gamma$. We also denote by $[x]$ the ray equivalence class of $x$. The diameter of a ray equivalence class is computed with respect to the graph metric. The vertex set of a ray equivalence class $[x]$ has a natural partition consisting of the two sets $[x] \cap \mathcal{K}(P)$ and $[x] \cap \mathcal{K}(Q)$, and using this partition $[x]$ is seen to be a bipartite graph.

An equivalence relation $\sim$ on $\mathbb{S}^{2}$ is said to be Moore-unobstructed if the quotient $\mathbb{S}^{2} / \sim$ is homeomorphic to $\mathbb{S}^{2}$. The following theorem is due to A. Epstein (see [36, Proposition 4.12]).

Proposition 4.20. Let $P$ and $Q$ be two anti-polynomials of equal degree $d \geq 2$ with connected and locally connected Julia sets. If the equivalence classes of $\sim_{\text {ray }}$ are ray-trees having uniformly bounded diameter, then $\mathbb{S}_{P, Q}^{2} / \sim_{\text {ray }}$ is Moore-unobstructed.

We remark that the proof of this theorem uses Moore's theorem. It is originally stated only for polynomials, but the proof extends identically to the case of anti-polynomials. There is also a partial converse of the above statement which we will not be using here.

For a critically fixed anti-polynomial $P$, we have the following description of the cut-points of $\mathcal{K}(P)$. The result directly follows from the dynamical correspondence between function kissing reflection groups and critically fixed anti-polynomials discussed in Subsection 4.3.

Lemma 4.21. Let $P$ be a critically fixed anti-polynomial. Then any cut-point of $\mathcal{K}(P)$ is eventually mapped to a repelling fixed point that is the landing point of a 2-cycle of external rays.

An extended external ray that contains a repelling fixed point of a critically fixed anti-polynomial is called a principal ray. The ray equivalence class of a principal ray is called a principal ray class. In our setting, the Moore obstructions can be detected simply by looking at these principal ray classes. 
Lemma 4.22. Let $P$ and $Q$ be two marked anti-polynomials of equal degree $d \geq 2$, where $P$ is critically fixed and $Q$ is postcritically finite and hyperbolic.

1. If a principal ray equivalence class of $\sim_{\text {ray }}$ contains more than one cut-point of $\mathcal{K}(P)$, it must contain a 2-cycle or a 4-cycle.

2. The equivalence $\sim_{\text {ray }}$ is Moore-obstructed if and only if some principal ray equivalence class contains a 2-cycle or a 4-cycle.

Proof. We first note that since the second iterate of a postcritically finite anti-polynomial is a postcritically finite holomorphic polynomial with the same Julia set, it follows from [31, §9, §19] that the Julia sets of $P, Q$ are connected and locally connected.

Let $x \in \mathcal{K}(P)$ be a repelling fixed point whose ray equivalence class $[x]$ contains more than one cut-point of $\mathcal{K}(P)$. Each point of $\mathcal{J}(P)$ is the landing point of one or two rays. Since $[x]$ is connected, there must be cut-points $w^{\prime}, z^{\prime} \in[x]$ in $\mathcal{K}(P)$ so that $w^{\prime} \sim_{\text {ray }} z^{\prime}$ with $w^{\prime}$ and $z^{\prime}$ having graph distance two. Let $\gamma$ be the union of the two rays realising the connection between $w^{\prime}$ and $z^{\prime}$. For some iterate $j$, we have from Lemma 4.21 that $(P \Perp Q)^{j}\left(w^{\prime}\right)$ and $(P \Perp Q)^{j}\left(z^{\prime}\right)$ are fixed points, which we denote $w$ and $z$ respectively. The graph distance between $w$ and $z$ is either 0 or 2 . In the former case, $w=z$, and since $Q$ is hyperbolic and hence a local homeomorphism on $\mathcal{J}(Q)$, we have that $(P \Perp Q)^{j}(\gamma)$ is a 2-cycle. Since $x$ is a fixed point, its ray class is forward invariant, so $(P \Perp Q)^{j}(\gamma)$ must be contained in $[x]$. This yields a 2-cycle in $[x]$.

Suppose now that $w \neq z$. There then exist two extended external rays $\alpha_{z}$ and $\alpha_{w}$ that land at $z$ and $w$ and share a common endpoint $v \in \mathcal{K}(Q)$. Let $\gamma$ be the concatenation of these two rays. Each of $\alpha_{z}, \alpha_{w}$ must have dynamical period 2 since they land at fixed cut-points of $P$. If $v$ is a fixed point, then the graph $\alpha_{w} \cup(P \Perp Q)\left(\alpha_{w}\right)$ is a cycle of length 2 for $\sim_{\text {ray }}$. If $v$ has period 2, then $\gamma \cup(P \Perp Q)(\gamma)$ is a cycle of length 4 for $\sim_{\text {ray }}$. As argued before, either of these cycles must be in $[x]$. This concludes the proof of statement 1.

If a principal ray equivalence contains a cycle, it is immediate that $\sim_{\text {ray }}$ is Moore-obstructed. Now assume that no principal ray equivalence class contains a 2-cycle or a 4-cycle. This means that the diameter of a principal ray equivalence class is bounded by 4 as there can be at most one cut-point of $\mathcal{K}(P)$ in this class. Any principal ray equivalence class must evidently be a tree.

Now let $z \in \mathcal{J}(P)$. If there is no cut-point in the equivalence class $[z]$, then $[z]$ is a tree and has diameter at most 2. Otherwise, by Lemma 4.21 , it is eventually mapped to a principal ray equivalence class. Since both $P$ and $Q$ are hyperbolic, the map $P \Perp Q$ is a local homeomorphism on $\mathcal{J}(P)$ and $\mathcal{J}(Q)$. Since all of the principal ray equivalence classes are trees, in particular, simply connected, any component of its preimage under $P \Perp Q$ is homeomorphic to it. Thus, by induction, we conclude that $[z]$ is a tree and has diameter at most 4. Now by Proposition 4.20, the mating is Moore-unobstructed.

We now prove that for a large class of pairs of anti-polynomials, the absence of Moore obstruction is equivalent to the existence of geometric mating.

Proposition 4.23. Let $P$ and $Q$ be marked anti-polynomials of equal degree $d \geq 2$, where $P$ is critically fixed and $Q$ is postcritically finite and hyperbolic. There is an anti-rational map that is the geometric mating of $P$ and $Q$ if and only if $\sim_{\text {ray }}$ is not Moore-obstructed.

Proof. The only if part of the statement is immediate. To prove the if part, we will first show that the absence of Moore obstruction can be promoted to the absence of Thurston obstruction. Note that the only case where $(P \Perp Q)^{2}$ has nonhyperbolic orbifold is when $P$ and $Q$ are both power maps, in which case the conclusion of the theorem is immediate. Thus, we assume for the remainder of the proof that $(P \Perp Q)^{2}$ has hyperbolic orbifold.

Suppose $(P \Perp Q)^{2}$ has a Thurston obstruction $\Gamma$. We may assume that $\Gamma$ is irreducible. Since $Q$ has no Thurston obstruction, there is some edge $\lambda$ in the Hubbard tree $H_{P}$ so that $\Gamma \cdot \lambda \neq 0$. Note that $\lambda$ is an irreducible arc system consisting of one arc, so it follows from [42, Theorem 3.9] that $\Gamma$ must also be a Levy cycle consisting of one essential curve $\gamma$. 

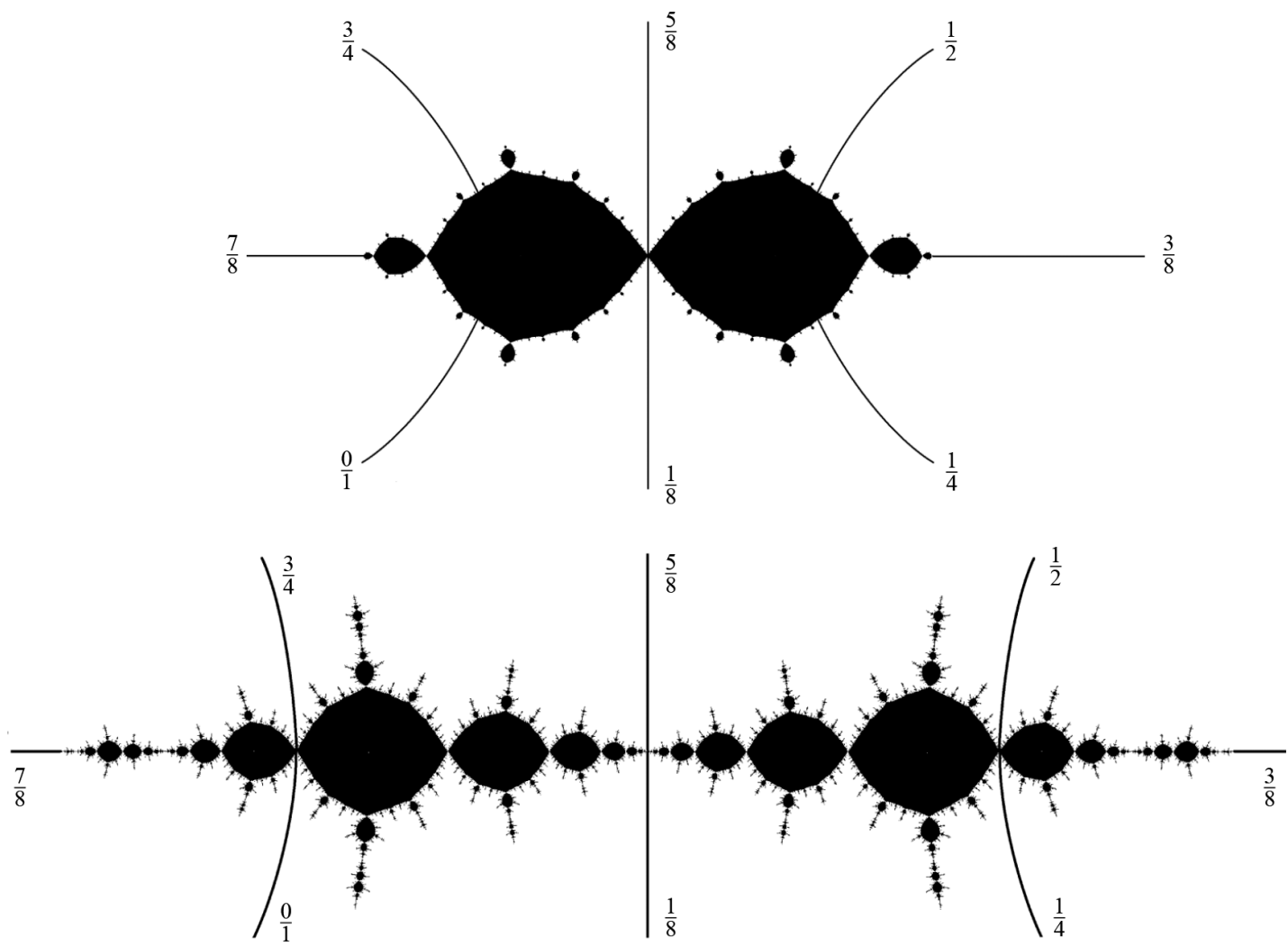

Figure 4.2. Two cubic anti-polynomials together with all rays that have period 2 or smaller. The first map is a critically fixed anti-polynomial so that the $1 / 8$ and 5/8 rays co-land. The second map is produced by tuning the first map with basilicas so that the pairs $(0 / 1,3 / 4)$ and $(1 / 2,1 / 4)$ co-land. The unique principal ray equivalence class has no cycle. Their mating is depicted below using the software [1].
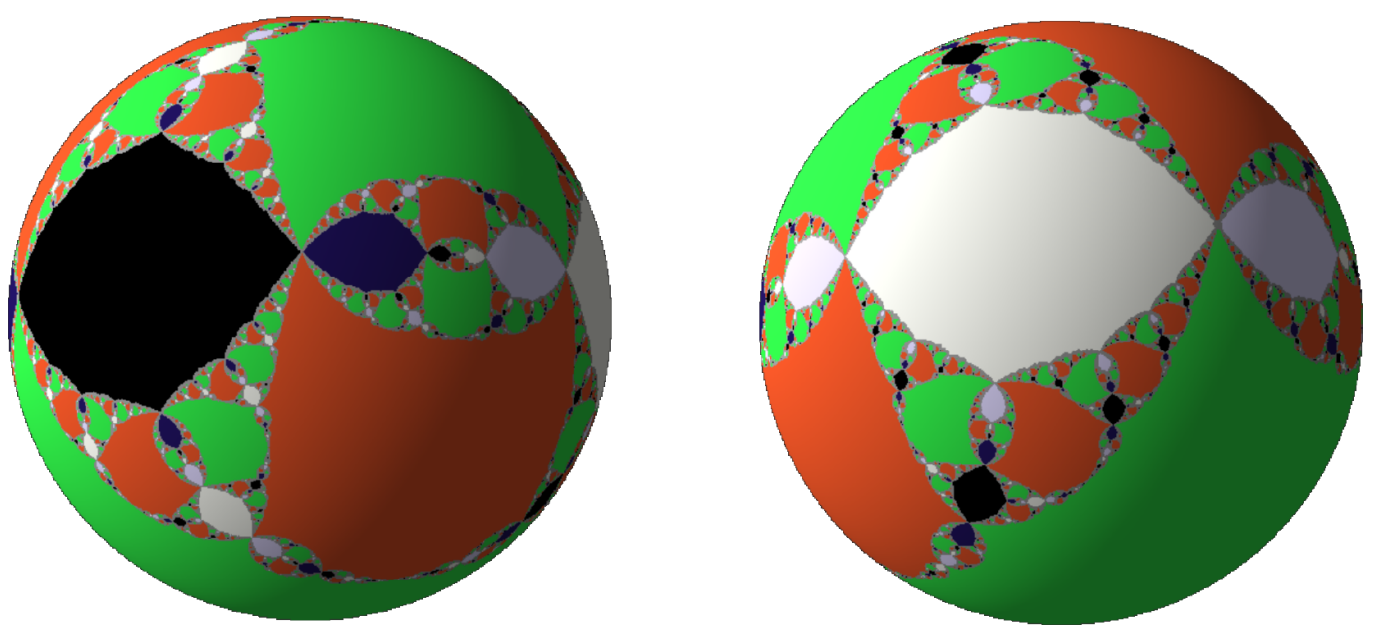

Since $P \Perp Q$ is hyperbolic, the Levy cycle is not degenerate (as defined in [42, §1]). Thus, there is a periodic ray class that contains a cycle [42, Theorem 1.4(2)]. By the hypothesis that there is no Moore obstruction, no such periodic ray class exists. This is a contradiction and so no Thurston obstruction exists 
for $(P \Perp Q)^{2}$. By Proposition 4.11, it follows that $P \Perp Q$ is also unobstructed and Thurston equivalent to a rational map.

Having shown that $P \Perp Q$ has no Thurston obstruction, the Rees-Shishikura theorem (which extends directly to orientation-reversing maps) implies that the geometric mating of $P$ and $Q$ exists [41, Theorem 1.7].

Recall that a pair of laminations on $\mathbb{S}^{1}$ for marked anti-polynomials is said to be nonparallel if they share no common leaf under the natural orientation-reversing identification of the two copies of $\mathbb{S}^{1}$. This is equivalent to saying that the ray equivalence classes contain no 2-cycle. We immediately have the following corollary from Lemma 4.22 and Proposition 4.23.

Corollary 4.24. A marked critically fixed anti-polynomial $P$ and a marked postcritically finite, hyperbolic anti-polynomial $Q$ are geometrically mateable if and only if the principal ray equivalence classes contain no 2-cycles or 4-cycles.

Two marked critically fixed anti-polynomials $P$ and $Q$ are geometrically mateable if and only if their laminations are nonparallel.

Proof. The first statement is immediate.

To see the second one, we note that according to Lemma 4.21, the lamination of a marked critically fixed anti-polynomial is generated by the period 2 cycles of rays landing at the repelling fixed points (which are cut-points). In light of this, it is easy to see that for two marked critically fixed antipolynomials, no principal ray equivalence class contains a 4-cycle. The second statement now follows from this observation and the first statement.

Acknowledgements. The authors thank Curt McMullen for useful suggestions. They also thank Mikhail Hlushchanka for stimulating discussions. The authors are grateful to the anonymous referee for their valuable input. The third author thanks the Institute for Mathematical Sciences and Simons Center for Geometry and Physics at Stony Brook University for their hospitality during part of the work on this project. The third author was supported by an endowment from Infosys Foundation and SERB research grant SRG/2020/000018.

Conflict of interest: None.

\section{References}

[1] L. Bartholdi, 'IMG', GAP package (2014). URL: https://github.com/laurentbartholdi/img.

[2] R. Bowen, C. Series, 'Markov maps associated with Fuchsian groups', Publ. Math. l'I.H.É.S. 50 (1979), 153-170.

[3] X. Buff, URL: https://www.math.univ-toulouse.fr/ buff/Symmetries/fichiers_pdf/patterns.pdf.

[4] K. Cordwell, S. Gilbertson, N. Nuechterlein, K. Pilgrim and S. Pinella, 'On the classification of critically fixed rational maps', Conform. Geom. Dyn. 19 (2015), 51-94.

[5] A. Douady, 'Systèms dynamiques holomorphies', Astèrisque 105(6) (1983), 39-63.

[6] A. Douady and J. H. Hubbard, 'A proof of Thurston's topological characterization of rational functions', Acta Math. 171 (1993), 263-297.

[7] P. Doyle and C. McMullen, 'Solving the quintic by iteration', Acta Math. 163 (1989), 151-180.

[8] P. Fatou, Notice sur les travaux scientifiques de M. P. Fatou (Astronome titulaire de l'observatoire de Paris, 1929), 5-29. https://www.math.purdue.edu/ eremenko/dvi/fatou-b.pdf.

[9] L. Geyer, 'Classification of critically fixed anti-rational maps (2020). URL: https://arxiv.org/abs/2006.10788v1.

[10] M. Heins, 'On a class of conformal metrics', Nagoya Math. J. 21 (1962), 1-50.

[11] M. Hlushchanka, 'Tischler graphs of critically fixed rational maps and their applications' (2019). URL: https://arxiv.org/abs/1904.04759v1.

[12] J. Hu, F. Jimenez and O. Muzician, 'Rational maps with half symmetries, Julia sets and Multibrot sets in parameter planes', Contemp. Math. 573 (2012), 119-146.

[13] A. Hatcher and W. Thurston, 'Moduli spaces of circle packings'. URL: https://pi.math.cornell.edu/ $\sim$ hatcher/Papers/CirclePacking.pdf.

[14] J. Hubbard, 'Matings and the other side of the dictionary', Ann. Fac. sci. Toulouse Math., Serie 621 (2012), 1139-1147.

[15] Y. Imayoshi and M. Taniguchi, An Introduction to Teichmüller Spaces (Springer, Tokyo, 1992).

[16] L. Keen, B. Maskit and C. Series, 'Geometric finiteness and uniqueness for Kleinian groups with the circle packing limit sets', J. Reine Angew. Math. 436 (1993), 209-219.

[17] A. Kontorovich and K. Nakamura, 'Geometry and arithmetic of crystallographic sphere packings', Proc. Natl. Acad. Sci. 116(2) (2019), 436-441. 
[18] A. Kontorovich and H. Oh, 'Apollonian circle packings and closed horospheres on hyperbolic 3 manifolds', J. Amer. Math. Soc. 24(3) (2011), 603-648.

[19] K. Lazebnik, N. Makarov and S. Mukherjee, 'Univalent polynomials and Hubbard trees', Trans. Amer. Math. Soc. 374 (2021), 4839-4893.

[20] K. Lazebnik, N. Makarov and S. Mukherjee, 'Bers slices in families of univalent maps', Math. Z. https://doi.org/10.1007/s00209-021-02871-y.

[21] S.-Y. Lee, M. Lyubich, N. G. Makarov and S. Mukherjee, 'Dynamics of Schwarz reflections: the mating phenomena', Preprint, 2018, https://arxiv.org/abs/1811.04979v2.

[22] S. Y. Lee and N. Makarov, 'Sharpness of connectivity bounds for quadrature domains', Preprint, 2014, https://arxiv.org/abs/1411.3415v1.

[23] R. Lodge, Y. Luo and S. Mukherjee, 'On deformation space analogies between Kleinian reflection groups and antiholomorphic rational maps', manuscript in preparation.

[24] R. Lodge, M. Lyubich, S. Merenkov and S. Mukherjee, 'On dynamical gaskets generated by rational maps, Kleinian groups, and Schwarz reflections', Preprint, 2019, https://arxiv.org/abs/1912.13438v1.

[25] A. Marden, 'The geometry of finitely generated Kleinian groups', Ann. Math. 99(3) (1974), 383-462.

[26] A. Marden, Hyperbolic Manifolds, an Introduction in 2 and 3 Dimensions (Cambridge University Press, Cambridge, 2016).

[27] B. Maskit, 'Parabolic elements in Kleinian groups', Ann. Math. 117(3) (1983), 659-668.

[28] C. McMullen, 'Iteration on Teichmüller space', Invent. Math. 99 (1990), 425-453.

[29] C. McMullen, 'The classification of conformal dynamical systems', in Bott, Hopkins, Jaffe, Singer, Stroock and Yau, editors, Current Developments in Mathematics (International Press, Cambridge, MA, 1995), 323-360.

[30] D. Meyer, 'Unmating of rational maps: sufficient criteria and examples', in Frontiers in Complex Dynamics, Vol. 51 of Princeton Math. Ser. (Princeton Univ. Press, Princeton, NJ, 2014), 197-233.

[31] J. Milnor, 'Dynamics in one complex variable', in Annals of Mathematics Studies vol. 160, third edn (Princeton University Press, Princeton, NJ, 2006), viii+304.

[32] M. Mj and C. Series, 'Limits of limit sets I', Geom. Dedicata 167 (2013), 35-67.

[33] J. Nielsen, 'Untersuchungen zur Topologie der geschlossenen zweiseitigen Flächen', Acta Math. 50 (1927), 189-358.

[34] H. Oh and N. Shah, 'Counting visible circles on the sphere and Kleinian groups', in C. Aravinda, F. Farrell and J. Lafont, editors, Geometry, Topology, and Dynamics in Negative Curvature (London Mathematical Society Lecture Note Series) vol. 425 (Cambridge University Press, Cambridge, 2016), 272-288.

[35] K. Ohshika, 'Geometrically finite Kleinian groups and parabolic elements', Proc. Edinb. Math. Society 41(1) (1998), 141159.

[36] C. Petersen and D. Meyer, 'On the notions of mating', Ann. Fac. Sci. Toulouse 21 (2012), 839-876.

[37] K. Pilgrim, 'Combinations of complex dynamical systems', Lecture Notes in Mathematics vol. 1827 (Springer-Verlag, Berlin, 2003), x+118. doi: $10.1007 / \mathrm{b} 14147$.

[38] K. Pilgrim and L. Tan, 'Combining rational maps and controlling obstructions', Ergod. Theory Dynam. Syst. 18 (1998), 221-245.

[39] M. Rees, 'Multiple equivalent matings with the aeroplane polynomial', Ergod. Theory Dynam. Syst., 30 (2010), $1239-1257$.

[40] O. Schramm, 'How to cage an egg', Invent. Math. 107 (1992), 543-560.

[41] M. Shishikura, 'On a theorem of M. Rees for matings of polynomials', in The Mandelbrot Set, Theme and Variations (Cambridge Univ. Press, Cambridge, 2000), 289-305.

[42] M. Shishikura and L. Tan, 'A family of cubic rational maps and matings of cubic polynomials', Exp. Math. 9(1) (2000), 29-53.

[43] D. Sullivan, 'Quasiconformal homeomorphisms and dynamics I: solution of the Fatou-Julia problem on wandering domains', Ann. Math. 122 (1985), 401-418.

[44] W. Thurston, 'The geometry and topology of 3-manifolds', Princeton Lecture Notes, 1978-1981.

[45] W. Thurston, 'Hyperbolic structures on 3-manifolds I: deformation of acylindrical manifolds', Ann. Math. 124(2) (1986), 203-246.

[46] D. Tischler, 'Critical points and values of complex polynomials', J. Complexity 5(4) (1989), 438-456.

[47] E. B. Vinberg and O. V. Shvartsman, 'Discrete groups of motions of spaces of constant curvature', in Geometry II. Encyclopaedia of Mathematical Sciences vol. 29 (Springer, Berlin, 1993).

[48] B. Wittner, On the Bifurcation Loci of Rational Maps of Degree Two, Ph.D. Thesis (Cornell University, 1988). 